\title{
April 2000 magnetic storm: Solar wind driver and magnetospheric response
}

\author{
K. Emilia J. Huttunen, ${ }^{1}$ Hannu E. J. Koskinen, ${ }^{1,2}$ Tuija I. Pulkkinen, ${ }^{2}$ Antti Pulkkinen, ${ }^{2}$ \\ Minna Palmroth, ${ }^{2}$ E. Geoffrey D. Reeves, ${ }^{3}$ and Howard J. Singer ${ }^{4}$ \\ Received 2 November 2001; revised 4 June 2002; accepted 26 June 2002; published 13 December 2002.
}

[1] On 4 April 2000, a coronal mass ejection (CME) took place close to the western limb of the Sun. The shock front of the CME hit the Earth's magnetosphere on 6 April. A strong interplanetary southward $B_{Z}$ event in the sheath region caused a magnetic storm that was the second strongest in the year 2000 if quantified by the peak of the Dst index. We have analyzed this sequence of events using observations of several spacecraft in the solar wind and at geostationary orbit as well as recordings from more than 80 magnetometer stations at latitudes higher than $40^{\circ} \mathrm{N}$. In the sheath region behind the shock, the interplanetary magnetic field had an intense and long-sustained southward magnetic field orientation, and the solar wind magnetic pressure was very large, which compressed the dayside magnetopause inside geostationary orbit for a period of more than 6 hours. We conclude that it was the fluctuating but strongly southward field accompanied by the high pressure that allowed for the exceptionally strong driving of magnetospheric activity. During the main phase of the storm, the magnetosphere and ionosphere were in highly perturbed states, with several activations all around the auroral region. Detailed analysis shows that many of these activations were not substorms, in the sense that they were not associated with poleward and westward electrojet/auroral enhancement or geostationary particle injections, but were directly driven perturbations due to variations in the solar wind features. In fact, it was found that the development of the entire storm was quite independent of substorm activations and injections. Instead, the ring current development was driven by the strong convection enhancements. During the storm, the geomagnetically induced currents were strongly enhanced during several periods. While some activations were associated with substorm onsets or electrojet enhancements, others were caused by extremely localized and short-lived electrojet activations. INDEX TERMS: 2788

Magnetospheric Physics: Storms and substorms; 2111 Interplanetary Physics: Ejecta, driver gases, and magnetic clouds; 2463 Ionosphere: Plasma convection; 2139 Interplanetary Physics: Interplanetary shocks; 1515 Geomagnetism and Paleomagnetism: Geomagnetic induction

Citation: Huttunen, K. E. J., H. E. J. Koskinen, T. I. Pulkkinen, A. Pulkkinen, M. Palmroth, E. G. D. Reeves, and H. J. Singer, April 2000 magnetic storm: Solar wind driver and magnetospheric response, J. Geophys. Res., 107(A12), 1440, doi:10.1029/2001JA009154, 2002.

\section{Introduction}

[2] The year 2000 provided several intense and interesting solar-terrestrial events. The preliminary (no pressure correction) Dst index reached levels below $-200 \mathrm{nT}$ three times, of which one exceeded $-300 \mathrm{nT}$. In particular, the so-called Bastille Day storm, initiated by a coronal mass ejection (CME) on 14 July, has received considerable interest (for preliminary information, see the ISTP Web page (http://

\footnotetext{
${ }^{1}$ Department of Physical Sciences, Theoretical Physics Division, University of Helsinki, Helsinki, Finland.

${ }^{2}$ Geophysical Research, Finnish Meteorological Institute, Helsinki, Finland.

${ }^{3}$ Los Alamos National Laboratory, Los Alamos, New Mexico, USA.

${ }^{4}$ NOAA Space Environment Center, Boulder, Colorado, USA.

Copyright 2002 by the American Geophysical Union. 0148-0227/02/2001JA009154\$09.00
}

www-istp.gsfc.nasa.gov/istp/events/2000july14/)). In this study we analyze a large storm during which the second lowest Dst value during the year 2000, $-288 \mathrm{nT}$ (with pressure correction $-314 \mathrm{nT}$ ), was reached. The storm occurred on 6-7 April 2000, when magnificent auroral displays were seen in Central Europe as the main phase of the storm took place in the time sector 18-01 UT.

[3] It is now well established that CMEs lie behind practically all large magnetospheric storms [Tsurutani et al., 1988; Gosling et al., 1991; Richardson et al., 2001]. However, there is a rich variety in the geoefficiency of various features associated with interplanetary manifestations of CMEs (ICMEs) and it can make a large difference whether the magnetosphere is hit by a slow ICME without an interplanetary shock, by an ICME with shock, or by the shock only when the ICME ejecta does not intercept the magnetosphere [Gosling et al., 1991; Huttunen et al., 2002]. 
[4] Finally, the orientation of the interplanetary magnetic field (IMF) plays a decisive role in the energy transfer from the solar wind to the magnetosphere and only events with prolonged and sufficiently strong southward IMF can drive intense magnetospheric storms. For example, Gonzalez and Tsurutani [1987] determined that IMF Z component less than $-10 \mathrm{nT}$ lasting more than 3 hours can be used as a predictor for the Dst index reaching values below $-100 \mathrm{nT}$.

[5] The storm on 6-7 April 2000, was an example of a magnetospheric storm driven by an intense southward IMF in the sheath region between the shock and the ICME. As discussed below, early on 7 April there were signatures of CME ejecta, but the observations suggest that the spacecraft hit only the flanks of the ejecta. In this paper we perform a thorough analysis of the solar wind driver of the storm based on three spacecraft (SOHO, ACE, and WIND) in the upstream solar wind, and one (GEOTAIL) in the dayside magnetosheath. Furthermore, the geostationary GOES-8 and GOES-10 satellites crossed the dayside magnetopause during the event, which allows a reliable confirmation of the magnetopause location determined using the empirical magnetopause model of Shue et al. [1998].

[6] We examine the magnetospheric response to the solar wind driver using observations in the magnetosphere and in the polar ionosphere. This includes a careful analysis of the major substorm-like activations using data from a large number of magnetometer stations all around the highlatitude northern auroral zone. Furthermore, we discuss the large geomagnetically induced current (GIC) observed in Finland during the storm main phase. A better understanding of the detailed connection between the features of ICMEs and their geomagnetic consequences is essential in developing reliable warning and forecasting systems for operators of ground-based energy distribution systems.

\section{Data and Methods}

[7] We have used observations from several spacecraft, both inside and outside the magnetosphere. Three spacecraft, SOHO, ACE, and WIND, were monitoring the solar wind upstream of the Earth. ACE and SOHO were both located near the L1 point more than $200 R_{E}$ from the Earth, and WIND was located, early on 6 April, around $64 R_{E}$ from the Earth, and was traveling toward the Earth. Solar wind data from ACE, due to its relatively steady position, have been used to analyze features of the intense southward magnetic field structure after the shock that caused the major magnetic storm. The WIND data, in turn, due to the closer location of the spacecraft to the Earth, have been used to connect the disturbances in the solar wind to magnetic disturbances in the Earth's magnetosphere and ionosphere and to estimate the energy input into the magnetosphere as well as the magnetopause location. GEOTAIL made observations in the magnetosheath, and traversed from the morning side magnetosphere through the subsolar magnetopause to the duskside magnetosphere during the early phases of the storm. Positions of these four spacecraft projected on the ecliptic plane on 6-7 April 2000 are shown in Figure 1. Their locations at the time of the shock arrival are marked by filled circles. We have also used data from five geostationary satellites, GOES-8, GOES-10,

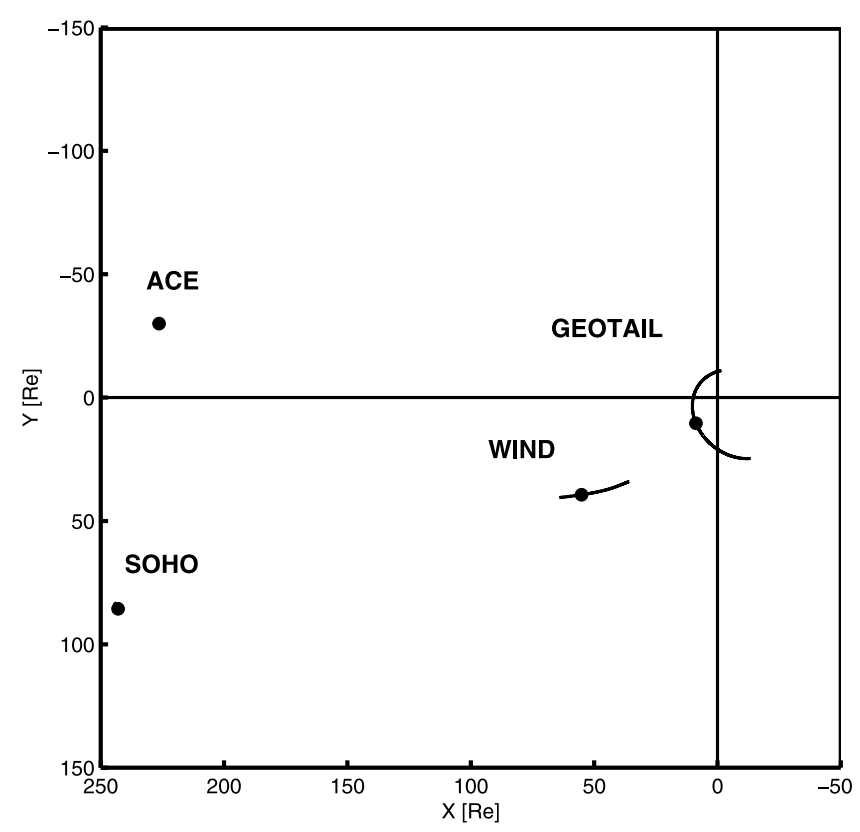

Figure 1. Position of SOHO, ACE, WIND, and GEOTAIL satellites in 6 and 7 April projected on the ecliptic plane. The satellite locations at the time of the shock observation are indicated by filled circles.

1989-046, 1991-080, and 1994-084, to study the dynamics of the inner magnetosphere.

[8] To determine the level of magnetic activity at the Earth we have used the Kp and Dst indices from the World Data Center C2 in Kyoto. The Dst index aims to measure the strength of the equatorial ring current, whereas $\mathrm{Kp}$ is a more global storm indicator often having significant contribution from the high-latitude auroral electrojets [e.g., Gonzalez et al., 1994]. We have also utilized 77 groundbased magnetometers all around the high-latitude auroral zone in order to study the global ionospheric response and to identify substorm signatures during the storm period.

\section{Storm Sequence From the Sun to the Earth 3.1. Solar Observations}

[9] The LASCO [Brueckner et al., 1995] C2 coronagraph onboard SOHO first observed a halo CME on 4 April 2000 at 1632 UT. This CME caused a strong magnetic storm at the Earth 2 days later. The initial observations were made after a 90 min data gap, when the leading edge had already left the $\mathrm{C} 2$ field of view. The coronagraph images show that most of the CME material was centered over the west limb. The plane-of-the-sky speed was reported to be $1188 \mathrm{~km} / \mathrm{s}$ (SOHO/LASCO CME catalog (http://cdaweb.gsfc.nasa. gov)). This CME event was associated also with other types of solar activity: GOES-8 satellite recorded a C9.7 class flare in the NOAA active region 8933 (N18W58) at 1524 UT. Also H-alpha images from Holloman AFB, New Mexico, showed a disappearance of a large filament preceding the CME onset. The filament was located near the northwest limb (N25W55) and it extended vertically along the surface of the Sun. The filament activation started at 1441 UT and continued until 1535 UT. 
April 6-7

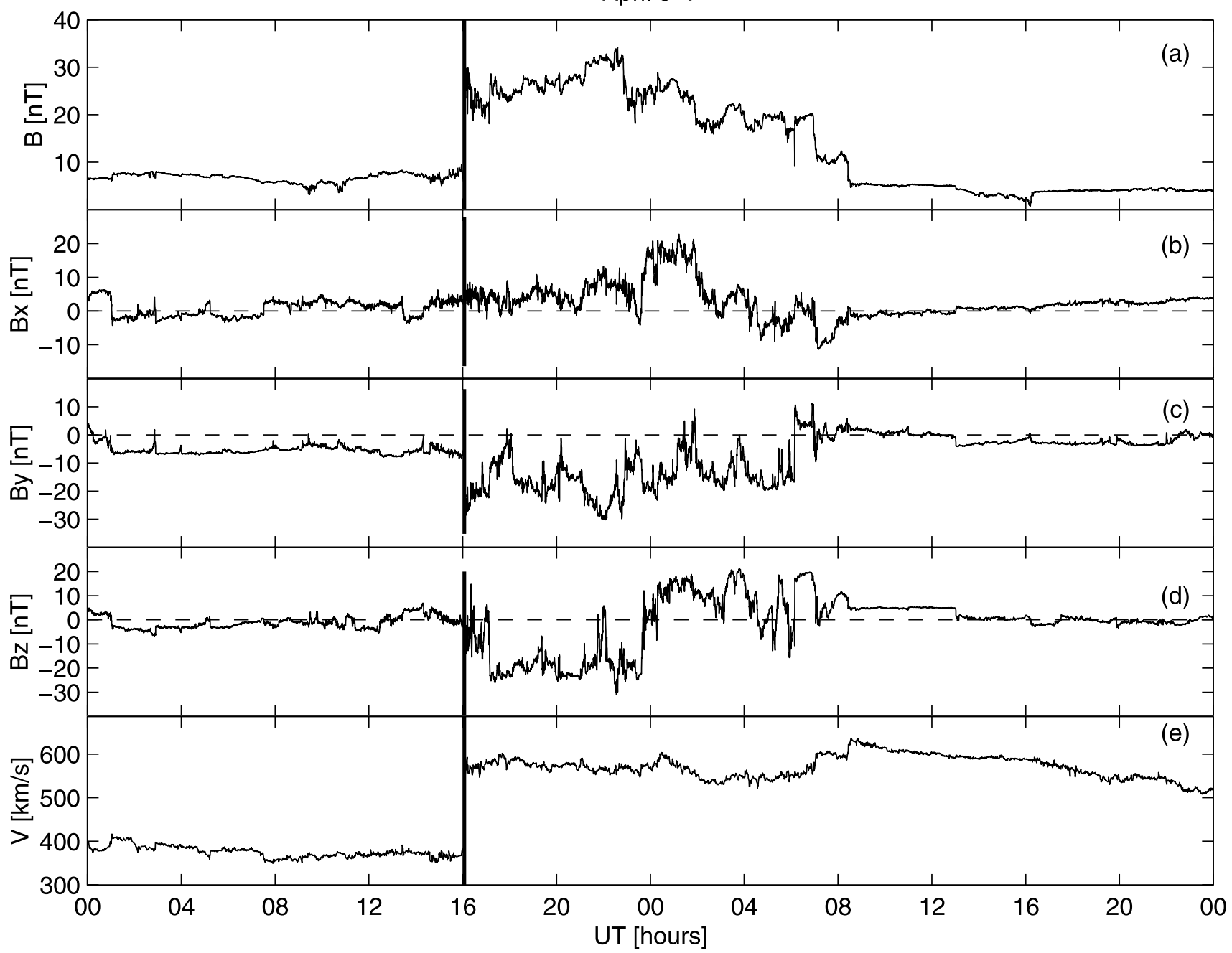

Figure 2. Solar wind parameters and geomagnetic indices for a 2 day interval from 6 to 8 April 2000 measured by ACE. The panels from top to bottom show the magnetic field intensity (a) and magnetic field components in the GSE coordinate system $(b-d)$, the ion speed (e), density (f), and temperature $(\mathrm{g})$, and the alpha-to-proton ratio and hourly average $\mathrm{O}^{+7} / \mathrm{O}^{+6}$ ratio $(\mathrm{h})$, proton beta (i), and dynamic pressure $(j)$. The dashed horizontal line in panel $g$ presents the temperature expected for normal solar wind expansion and the solid horizontal lines in panels $\mathrm{i}$ and $\mathrm{j}$ show limit values for CME-associated solar wind plasma.

[10] The first signature of this solar event near 1 AU was observed soon after the flare when a solar particle event was recorded. For example, GOES-8 measured the increase in $>10 \mathrm{MeV}$ protons starting around 1600 UT on 4 April 2000. Continuing acceleration of particles at the interplanetary shock maintained the intensities at an enhanced level until the shock arrival at 1 AU on 6 April.

\subsection{Solar Wind Observations}

[11] The strong interplanetary shock was first observed by the CELIAS instrument onboard SOHO at 1601 UT on 6 April. Shortly after this, the shock was recorded by ACE at 1604 UT and at 1632:28 UT by WIND. Finally it was detected at 1641:30 UT by GEOTAIL located in the magnetosheath. Figure 2 shows the ACE measurements for a 2 day period from 6 to 8 April. Shown in Figure 2 are the solar wind magnetic field intensity (a), magnetic field components in the GSE coordinate system $(b-d)$, solar wind speed (e), density (f) and temperature (g) as well as the solar wind alpha to proton ratio and the $\mathrm{O}^{+7} / \mathrm{O}^{+6}$ ratio divided by $10(\mathrm{~h})$. We have also calculated the proton beta (i) and dynamic pressure (j). The shock is clearly identifiable as a distinct jump in the solar wind parameters at 1604 UT. Before the shock, the solar wind was flowing at about $380 \mathrm{~km} / \mathrm{s}$; at the shock the solar wind speed increased suddenly by about 200 to almost $600 \mathrm{~km} / \mathrm{s}$. Simultaneously the ion density increased from 7 to about $22 \mathrm{~cm}^{-3}$, and the magnetic field intensity from 8 to $28 \mathrm{nT}$. The upstream Alfvén speed was $64 \mathrm{~km} / \mathrm{s}$ and the downstream Alfvén speed $128 \mathrm{~km} / \mathrm{s}$. The distance between ACE and WIND in the X-direction was $171 R_{E}$ and the time difference between the shock observations was about $28.5 \mathrm{~min}$. If the shock propagation is assumed to have been in the X-direction, this would yield an average shock speed of $638 \mathrm{~km} / \mathrm{s}$. 


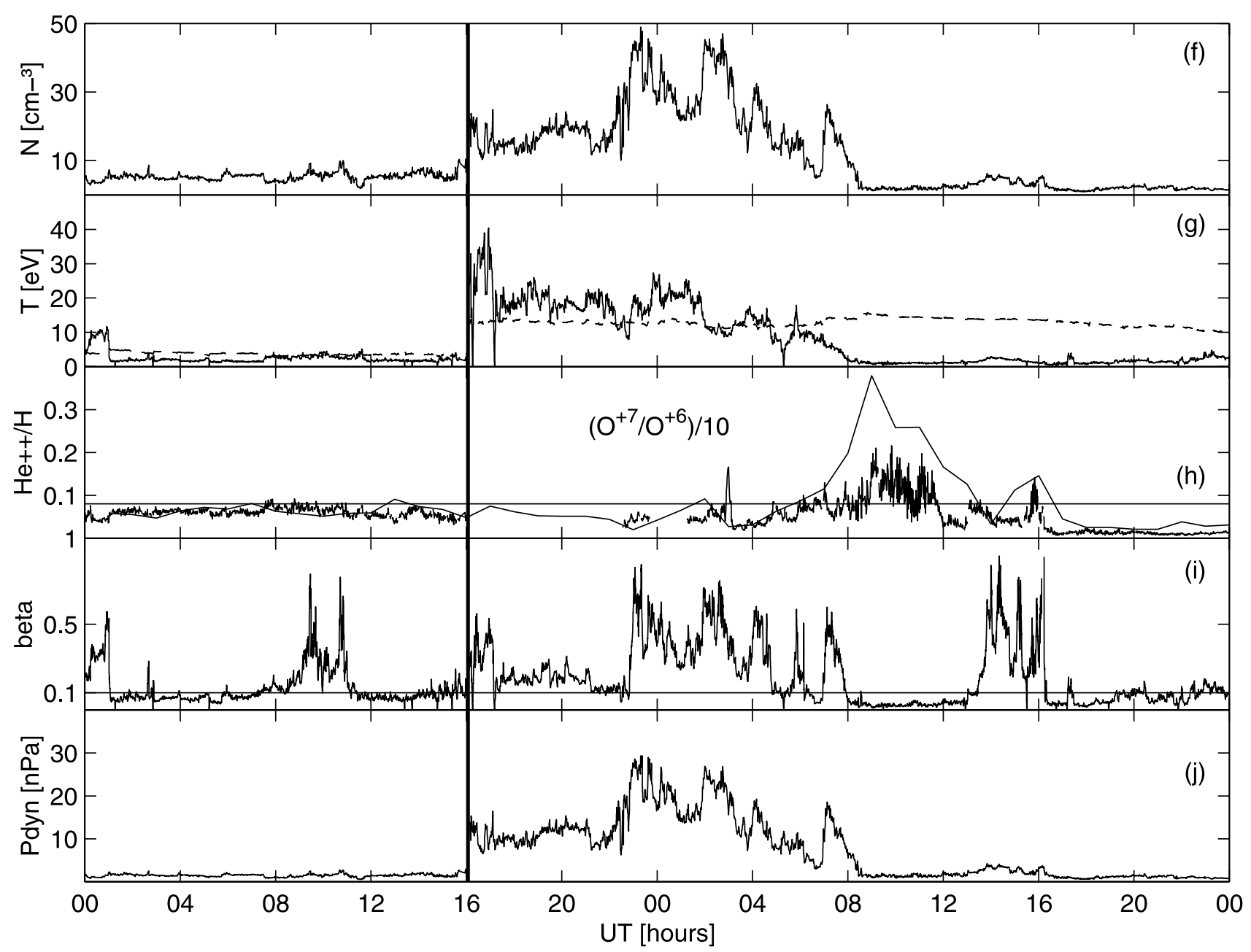

Figure 2. (continued)

[12] The direction of the magnetic field displayed irregular behavior both upstream and downstream of the shock. Also velocity downstream of the shock had irregular behavior. Thus, the shock normal direction $\left(\mathbf{n}_{\mathbf{s h}}\right)$ could not be determined very accurately. We have applied different techniques [e.g., Tsurutani and Lin, 1985] for different upstream and downstream intervals using data from WIND and ACE spacecraft. The velocity coplanarity theorem could not be used for ACE data as there was about a 10 min data gap in ACE solar wind measurement upstream of the shock. Depending on the method used and selected intervals quite different shock normal orientations are found. We have applied the magnetic coplanarity theorem $\mathbf{n}_{\mathbf{s h}} \|\left(\mathbf{B}^{\mathbf{u}}-\mathbf{B}^{\mathbf{d}}\right) \times\left(\mathbf{B}^{\mathbf{u}} \times \mathbf{B}^{\mathbf{d}}\right)$ where $u$ denotes upstream values and $d$ downstream values of the shock for ACE data, which was available with the highest cadence. This yields shock normal orientation $(-0.85,0.13,-0.52)$ in solar ecliptic coordinates or the longitudinal angle $\phi=171^{\circ}$ and latitudinal angle $\theta=-31^{\circ}$. The shock angle $\theta_{B n}$, i.e., the angle between the shock normal and the upstream magnetic field direction was $85^{\circ}$ defining the shock as quasi-perpendicular. The shock speed can be determined from conservation of the mass flux. Along the shock normal it is obtained from $\mathbf{V}_{\mathbf{s h}}=\mathbf{n}_{\mathbf{s h}} \cdot\left(\left(\rho^{\mathbf{u}} \mathbf{V}^{\mathbf{u}}-\rho^{\mathbf{d}} \mathbf{V}^{\mathbf{d}}\right) /\left(\rho^{\mathbf{u}}-\rho^{\mathbf{d}}\right)\right)$.
The chosen shock normal direction gives the shock propagation speed $635 \mathrm{~km} / \mathrm{s}$ along the shock normal which is consistent with the value calculated above and the Alfvénic Mach number 5.1. The shock normal direction is consistent with the CME originating from northern solar hemisphere, but the shock longitudinal angle is not that large as would be expected from the western source of the CME. From WIND measurements we obtain the shock normal direction $(\phi, \theta)=\left(169^{\circ},-48^{\circ}\right)$ using magnetic coplanarity theorem and $(\phi, \theta)=\left(170^{\circ},-44^{\circ}\right)$ using velocity coplanarity theorem $\left(\mathbf{n}_{\text {sh }} \|\left(\mathbf{B}^{\mathbf{u}}-\mathbf{B}^{\mathbf{d}}\right) \times\left(\left(\mathbf{V}^{\mathbf{u}}-\mathbf{V}^{\mathbf{d}}\right) \times \mathbf{B}^{\mathbf{u}}\right)\right)$. These values agree substantially well with the direction obtained from ACE but they give too slow shock velocity (about 560 $\mathrm{km} / \mathrm{s}$ ). Besides of the irregular behavior of the magnetic field and velocity, the magnetic coplanarity theorem is not reliable for near-perpendicular shocks. The shock normal determination could be improved by solving the nonlinear least squares Rankine-Hugoniot equations [e.g., Viñas and Scudder, 1986], but as the solar wind data needed for this method was available only from WIND with low cadence and the shock orientation is not the main concern of our paper this is not discussed further. The transfer time of first C2 CME observation to the shock observation at ACE was approximately 47.5 hours. However, it should be noted that 
coronagraph images are associated with the driver gas not the shock front.

[13] The IMF Z-component upstream of the shock was slightly negative, decreasing after the shock to values below $-10 \mathrm{nT}$. About 1 hour after the shock, the magnetic field turned strongly southward $\left(B_{Z}<-20 \mathrm{nT}\right)$ and the solar wind density and temperature decreased. $B_{Z}$ remained below $-20 \mathrm{nT}$ for about 7 hours and turned abruptly to northward orientation before 24 UT on 6 April 2000. After the shock the magnetic field intensity was high, over $30 \mathrm{nT}$, but its direction fluctuated rapidly. The IMF $X$-component was mainly positive and the $Y$-component mainly negative but they both showed a complex behavior, especially $B_{Y}$ which had long amplitude fluctuations. Slow, monotonic rotation of the magnetic field, which is the signature of an ICME exhibiting flux rope topology, commonly a called magnetic cloud [Burlaga, 1988], was not detected. Instead, $B_{Z}$ jumped rapidly from south to north.

[14] The solar wind speed at the edge of the high- $B$ structure was $570 \mathrm{~km} / \mathrm{s}$, and the speed profile stayed relatively flat. The solar wind density (Figure 2f) was clearly enhanced with respect to the ambient value throughout the high- $B$ structure. Initially, the density was between 10 and $20 \mathrm{~cm}^{-3}$, but about 1 hour before the $B_{Z}$ northward turning the density increased and had two larger enhancements reaching almost $50 \mathrm{~cm}^{-3}$. Solar wind temperature was also unusually high with a maximum value of about $3 \times 10^{5} \mathrm{~K}$. The dashed line in Figure $2 \mathrm{~g}$ shows the expected temperature $\left(T_{e x}\right)$ from the correlation of the solar wind speed and proton temperature for normal solar wind expansion [Richardson and Cane, 1995]. Unusually low temperatures are characteristic of ICMEs, and the proton temperature $\left(T_{p}\right)$ is often significantly depressed relative to the expected temperature $\left(T_{p} / T_{e x} \leq 0.5\right)[$ Richardson and Cane, 1995]. In this case the measured proton temperature well exceeded the expected temperature. Unusually high ratios of ionized helium number density to proton number density $\left(n_{\alpha} / n_{p}>0.08\right)$ have been shown to be present in many ICME events before, during, or after the ICME [Borrini et al., 1982]. Together with the $n_{\alpha} / n_{p}$ ratio in Figure $2 \mathrm{~h}$ is plotted the hourly average of the solar wind $\mathrm{O}^{+7} / \mathrm{O}^{+6}$ density ratio divided by 10 . Within slow-speed solar wind the $\mathrm{O}^{+7} / \mathrm{O}^{+6}$ ratio has high variability which distinguishes different sources of slow solar wind [Zurbuchen et al., 2000] and a high ratio of $\mathrm{O}^{+7} / \mathrm{O}^{+6}$ has been related to ICMEs having magnetic cloud topology [Henke et al., 1998]. The interval of the high helium abundance and enhanced $\mathrm{O}^{+7} / \mathrm{O}^{+6}$ ratio did not occur until $06 \mathrm{UT}$ on 7 April. Counterstreaming suprathermal electrons are some of the most commonly used ICME signatures [e.g., Gosling, 1990; Zwickl et al., 1983] interpreted to be indicative of a closed magnetic field configuration with field lines either attached to the Sun at both ends or forming closed loops. During the high- $B$ structure, there was no evidence of counterstreaming electrons in WIND 3D plasma summary plots (not shown).

[15] The solar wind dynamic pressure (Figure 2i) was $2 \mathrm{nPa}$ before the shock and increased to $10 \mathrm{nPa}$. The pressure increased further with time and reached values as high as $25 \mathrm{nPa}$. The proton beta is shown in Figure $2 \mathrm{j}$ with the solid line marking the value 0.1 . Usually beta is less than 0.1 in the ICME-associated solar wind plasma [Burlaga et al., 1981].
In this case, just after the shock, proton beta was over 0.5 and it dropped to about 0.2 at the time of the $B_{Z}$ decrease. Later, the beta values rose again due to the increased particle pressure.

[16] The duration of the high- $B$ structure after the shock was 16 hours giving a radial extent of $0.22 \mathrm{AU}$ using the average speed of $570 \mathrm{~km} / \mathrm{s}$. Following the high- $B$ structure, Figure 2 shows a period of fast flow with low magnetic field intensity (about $5 \mathrm{nT}$ ) starting around 0830 UT on 7 April 2000. This flow was not a corotating stream [Smith and Wolf, 1976] as the temperature was very low. Many signatures of the ICME-related plasma [e.g., Gosling, 1990] were detected a few hours before and during the fast flow. On 7 April there was a discontinuity at 0609 UT when the longitude angle rotated about $170^{\circ}$ and the latitude angle about $80^{\circ}$. Solar wind plasma parameters and magnetic field intensity did not change in magnitude and the magnetic field normal to the discontinuity boundary had a nonzero value suggesting a rotational discontinuity. Just after the discontinuity, the magnetic field became smoother and there was an increase above the CME-related plasma limit in the ratio of $n_{\alpha} / n_{p}(>0.08)$ as well as in the $\mathrm{O}^{+7} / \mathrm{O}^{+6}$ ratio $(>1.0)$. However, magnetic field strength decreased within 2 hours to as low as $5 \mathrm{nT}$. At 08 UT on 7 April plasma beta dropped to very low values $(<0.1)$ and coincidentally the temperature was much lower than would be expected during such high solar wind speed plasma [Richardson and Cane, 1995]. Also WIND 3D plasma summary plots (not shown) showed a counterstreaming suprathermal electron event starting around 08 UT on 7 April. Based on the magnetic field observations and other ICME signatures described above, we suggest that the boundary between the sheath region plasma and the ICME plasma was around 06 UT on 7 April. Thus, the ICME arrived about 12 hours after the shock. The transit time for the ICME from the first $\mathrm{C} 2$ observation is 61.5 hours, giving the average transit speed of $668 \mathrm{~km} / \mathrm{s}$.

[17] In conclusion, the geoeffective structure after the shock (1604 UT on 6 April to 06 UT on 7 April 2000), that is our main interest, was certainly not expanding and fails to fulfill the criteria for an ICME. The rapidly varying magnetic field direction, high temperature and density are rather characteristic of the sheath region [Tsurutani et al., 1988], i.e., the piled-up solar wind between the shock and the CME ejecta. After the sheath region, starting around 06 UT on 7 April, there were many ICME signatures present but it was not evident where the boundary of ICME plasma was exactly located. The identification of an ICME is not unambiguous as a single ICME seldom exhibits all ICME signatures and different features do not necessarily occur at the same time and during the whole passage of the ICME [e.g., Gosling, 1990; Zwickl et al., 1983]. For this case the identification was further complicated as the spacecraft (and the Earth) most likely hit the ejecta far from its center where the magnetic field and solar wind signatures are not so distinct as near the center of the ICME.

[18] Magnetic clouds are in many cases associated with disappearing filaments and the orientation of magnetic clouds axes are found to generally coincide with the orientation of the filament axis [Bothmer and Schwenn, 1994; Marubashi, 1997] (i.e., filaments that are elongated horizontally along the surface of the Sun produce magnetic clouds that have their axes lying in the ecliptic plane and 


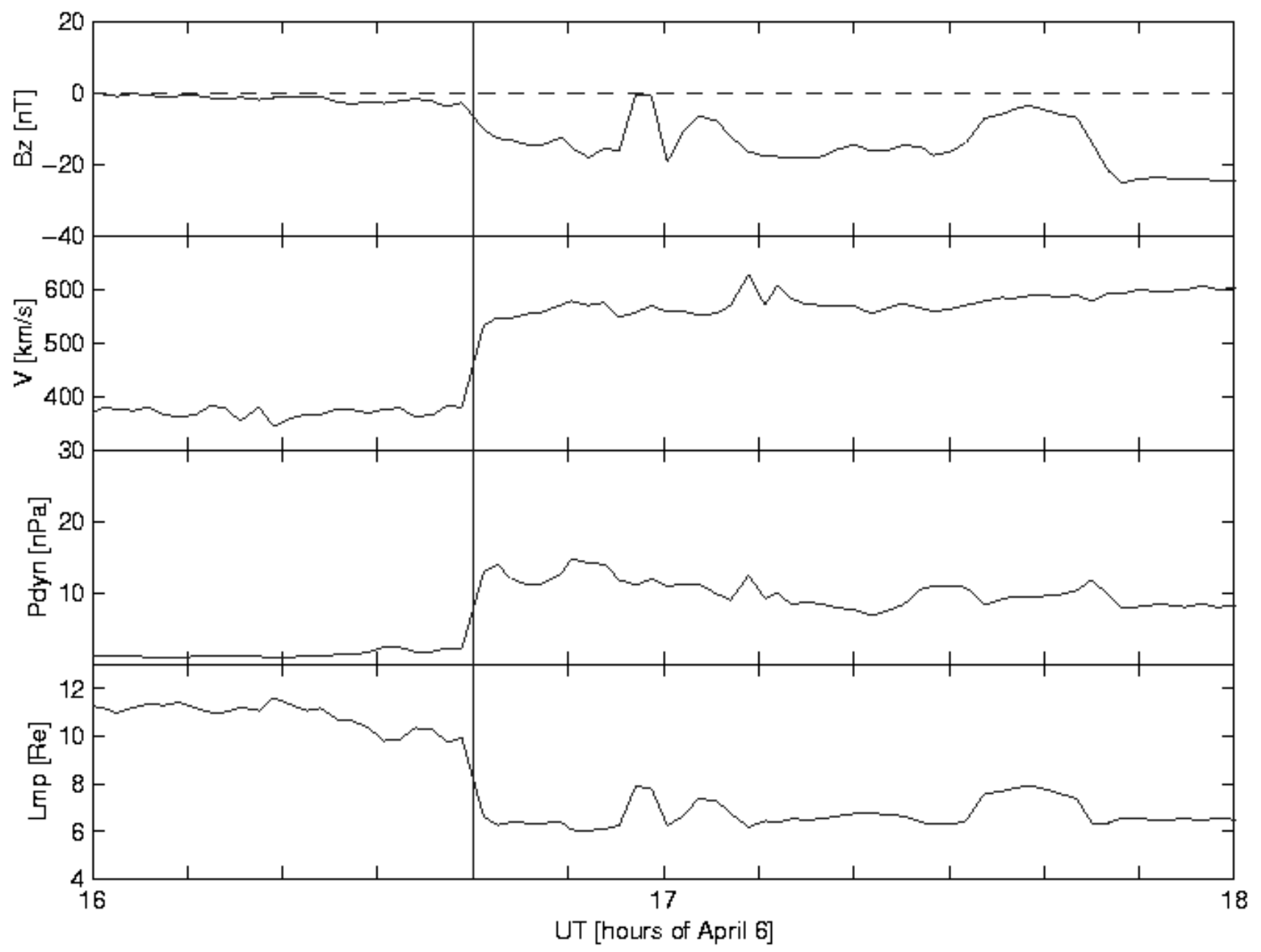

Figure 3. Magnetic field Z-component in GSM coordinates (a), solar wind speed (b), dynamic pressure from WIND data (c), and calculated subsolar distance to the magnetopause (d). WIND data have been shifted $12 \mathrm{~min}$ before and $8 \mathrm{~min}$ after the shock arrival at 1640 UT (indicated by the vertical line). The overlapping points have been omitted.

vertical filaments produce CMEs that have their axis perpendicular to the ecliptic plane). Enhanced $\mathrm{O}^{+7} / \mathrm{O}^{+6}$ and $n_{\mathrm{\alpha}} / n_{p}$ ratio, low beta and temperature as well as counterstreaming electrons are characteristic to magnetic clouds [e.g., Zwickl et al., 1983; Gosling, 1990; Henke et al., 1998]. Furthermore as the CME on 4 April 2000 was associated with the filament disappearance it is likely that the structure spacecraft encountered on 7 April 2000 at about 6 UT was a part of the ICME with magnetic cloud structure. As the spacecraft hit only the flanks of this magnetic cloud, we cannot determine its orientation from magnetic field data. However, $\mathrm{H}_{\mathrm{\alpha}}$-images of the associated filament show that it was vertically elongated along the surface of the Sun, from which we can infer that the axis of the magnetic cloud likely had high inclination relative to the ecliptic plane. It should be noted that the shock surface is not a planar structure but curved around the ejecta and thus in this case the axis of the magnetic cloud should have had a large positive inclination relative to ecliptic to explain the obtained large negative latitudinal angle of the shock normal. Furthermore, as discussed in section 3.3 measurements from geostationary orbit (GOES8 and GOES-10) showed that the magnetosphere was more compressed at the dawnside. Based on the observations described above we suggest the scenario where the magnetic cloud, highly inclined relative to ecliptic, swept the Earth's magnetosphere from above and dawnside. Also the $Z$-component of the velocity had large negative values (down to $-120 \mathrm{~km} / \mathrm{s}$ in GSE coordinate system) downstream of the shock consistent with the direction of the plasma flow bending around the ejecta having the above mentioned orientation.

\subsection{Arrival at Earth}

[19] Figure 3 shows the IMF Z-component, the solar wind speed and the dynamic pressure as measured by WIND and the subsolar distance to the magnetopause as computed from the Shue et al. [1998] model. As discussed in the previous section, the shock was detected at the location of WIND at 1632 UT and in the magnetosheath by GEOTAIL at $1640 \mathrm{UT}$. This is consistent with the propagation time of $8 \mathrm{~min}$ of the shock calculated from WIND observations. 
Note that the structures in front of the shock arrived at a lower speed with a transit time of $12 \mathrm{~min}$. In Figure 3 we have shifted the data before the shock by $12 \mathrm{~min}$ and after the shock by $8 \mathrm{~min}$ to represent the plasma conditions at the dayside magnetopause. The overlapping data points were omitted as physically they were hidden inside the shock structure.

[20] The empirical model by Shue et al. [1998] gives the magnetopause location as a function of IMF $B_{Z}$ and the solar wind dynamic pressure. We have used the WIND measurements and to calculate the dynamic pressure from proton density we have assumed the alpha-to-proton ratio to be $4 \%$. Before the shock arrival, the subsolar magnetopause was close to its quiet-day position around $11 R_{E}$. The magnetopause started to move inward before the shock arrival. This was caused by both the decrease of $B_{Z}$ and the density increase in the shock foot region. The magnetopause was compressed close to the geostationary orbit at about 1644 UT. The magnetosphere remained in a very compressed state until the early hours of 7 April. The closest subsolar standoff distance was about $5.5 R_{E}$ at $2351 \mathrm{UT}$.

[21] In Figure 4 we compare geostationary magnetic field observations to the magnetopause position as predicted by the Shue et al. [1998] model. The upper two panels show the magnetic field observations from GOES-10 and the calculated distance to the magnetopause in the direction of the spacecraft $\left(R\right.$, thick line) and to the subsolar point $\left(R_{0}\right.$, thin line). The two lower panels show the same parameters for GOES-8. Both GOES-8 and GOES-10 observed the shock hitting the magnetopause at 1640 UT as a rapid jump in the magnetic field intensity. At this time both spacecraft were well inside the magnetosphere.

[22] GOES-10, located at east geographic longitude $225^{\circ}$, just west of the US, moved during the magnetospheric compression from the prenoon to the afternoon sector. It crossed the magnetopause at $1801 \mathrm{UT}$, as shown by the rapid turning of the magnetic field north component to a southward orientation. At the location of the satellite, the model magnetopause distance was still $7.5 R_{E}$, indicating that the real compression was much stronger than that predicted by the model. GOES-10 passed the local noon at 2100 UT. After 2155 UT the magnetopause flapped back and forth over the satellite several times, but during 23200023 UT the satellite was again in the magnetosheath.

[23] GOES- 8 , located at east longitude $285^{\circ}$ above the US East coast, was in the prenoon magnetosphere before the arrival of the shock. At the time of the beginning of the magnetospheric compression the spacecraft was in the noon sector, as nicely illustrated by the coincidence of $R$ and $R_{0}$ in the lowermost panel of Figure 4. According to the magnetic field observations the magnetopause made a short passage over GOES-8 at 1729 UT and was steadily inside its orbit 1756-1839 UT.

[24] Additional information about the timing of the shock hitting the magnetosphere can be inferred from Figure 5, where we have calculated the auroral electrojet index based on 77 magnetometers at latitudes higher than $40^{\circ}$ (discussed in more detail in section 3.4). Furthermore, data from two individual magnetometers are shown from Baker Lake $\left(64.4^{\circ} \mathrm{N}, 264^{\circ} \mathrm{E}\right)$ and Oulujärvi $\left(64.5^{\circ} \mathrm{N}, 27.2^{\circ} \mathrm{E}\right)$. All these data show a clear global storm sudden commencement (SSC) at $1641 \mathrm{UT}$.
[25] Within the 1 min accuracy of magnetometer data both in space and on ground one gets a consistent picture of the arrival of the interplanetary shock effects at the magnetopause. By 1641 UT the change in the dayside magnetopause current system was observable all around the globe.

\subsection{Magnetospheric Activity}

\subsubsection{Global Activity and Energy Input}

[26] In order to estimate the total energy input to the magnetosphere we have calculated the epsilon parameter using measurements from the WIND satellite. Figure $6 \mathrm{a}$ repeats the IMF $B_{Z}$ as measured by WIND and shifted 8 min to the magnetopause (here we have not used the longer delay before the shock as it is not relevant for the analysis). Figures $6 \mathrm{~b}$ and $6 \mathrm{c}$ present the $\epsilon$-parameter $\left(\epsilon=10^{7} V \mathrm{~B}^{2} l_{0}^{2}\right.$ $\sin ^{4}(\theta / 2)$, using SI units) which is a function of the solar wind velocity $V$ and the IMF intensity $B$ and direction $(\tan \theta$ $=B_{Y}\left(B_{Z}\right)\left[\right.$ Akasofu, 1979, 1981]. $l_{0}=7 R_{E}$ is an empirical parameter determined to fit the average energy input to the average estimated output. The large variations in the magnitudes are the reason for showing the $\varepsilon$ parameter both in logarithmic and linear scales. As the IMF was southward at the shock arrival, the energy input to the magnetosphere increased immediately after the shock passage to above $5 \times 10^{12} \mathrm{~W}$, although the largest energy input started only around 1750 UT when the IMF turned strongly southward. The maximum value of epsilon, $1.3 \times 10^{13} \mathrm{~W}$, was reached at 2311 UT. The interplanetary $B_{Z}$ reached its minimum value $-33 \mathrm{nT}$ (in the GSM coordinate system) at about $2312 \mathrm{UT}$. The IMF $B_{Z}$ stayed significantly southward $(<-20 \mathrm{nT})$ until 0016 UT on 7 April, with $\epsilon$ well above typical storm threshold values of $10^{12} \mathrm{~W}$ [Akasofu, 1981].

[27] The dynamic pressure increased to about $10 \mathrm{nPa}$ at the shock arrival, and stayed at that level until about 23 UT (Figure 6d). Just after the $B_{Z}$ minimum, the dynamic pressure increased to near $20 \mathrm{nPa}$ showing oscillatory behavior with a period of about $10 \mathrm{~min}$. The global magnetic activity is described in terms of the SYM H, which is a highresolution (1 min) Dst-like index (also available at WDC2, Kyoto). The positive jump in the SYM H index was not very large at the shock arrival, as the southward IMF initiated dayside magnetic field erosion simultaneously with the pressure increase. Immediately following the shock passage, the SYM H index started a steady decrease which continued until about 0015 on 7 April (Figure 6e). The storm recovery phase began after about 01 UT on 7 April, with increasing Dst (and SYM H) values. The high dynamic pressure during the entire storm main phase would make the measured SYM $\mathrm{H}$ values even lower, if they were pressure corrected [Burton et al., 1975]. The pressure-corrected Dst reached a minimum value of $-314 \mathrm{nT}$ at $01 \mathrm{UT}$ on 7 April, 2 hours after the $B_{Z}$ minimum.

[28] The Kp index (not shown) reached a storm value of 7- during 15-18 UT. Kp was 8+ during 18-24 UT and finally reached its maximum value $9-$ during $00-03$ UT on 7 April.

[29] Figure 6f shows the IL index, which is an electrojet index created from the IMAGE magnetometer network stations [Tanskanen et al., 2001]. As IMAGE was in the night sector (from around 19 MLT at 1641 UT to around 0430 MLT at $0200 \mathrm{UT}$ ), it was well positioned to measure 


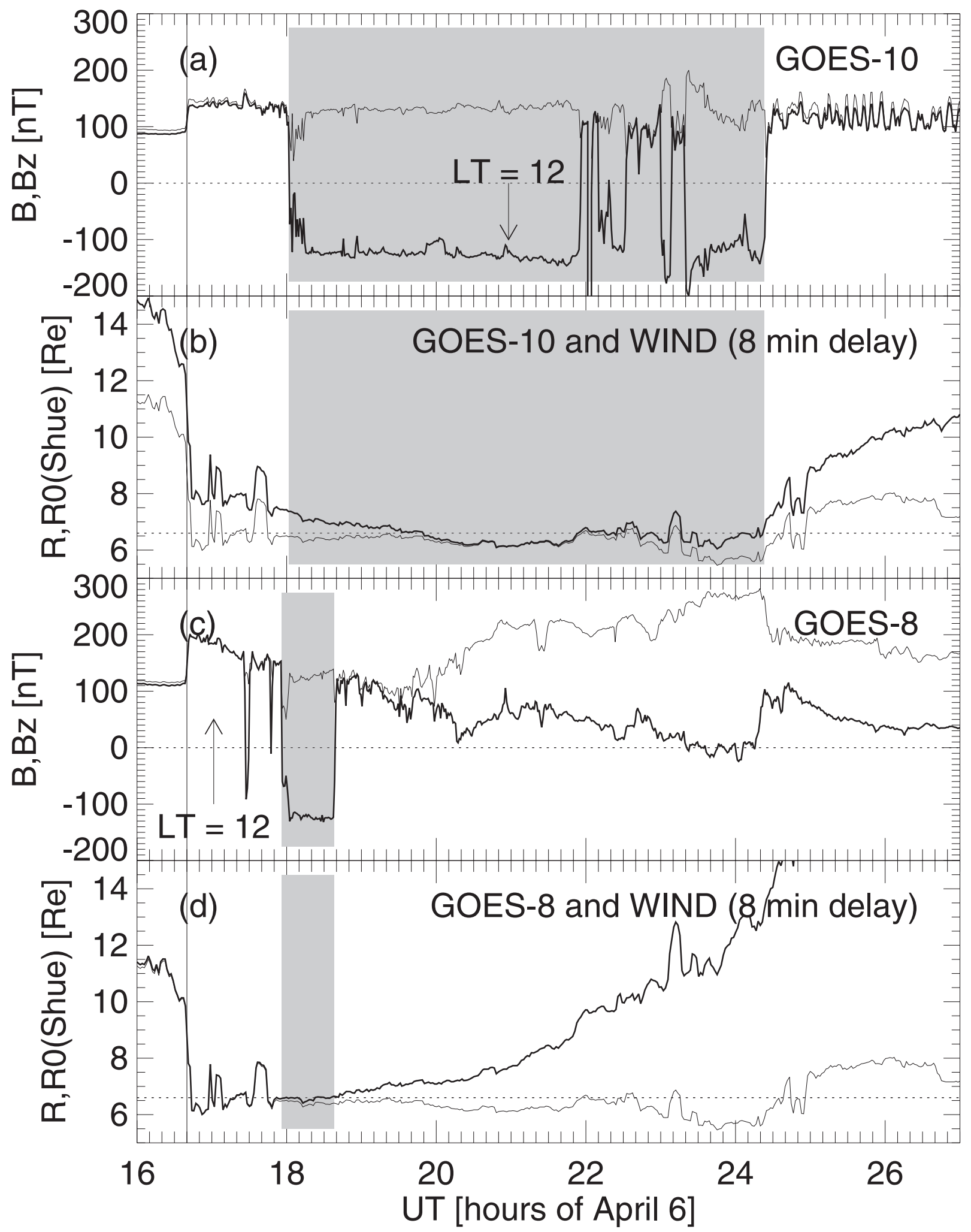

Figure 4. (a) Magnetic field observations at GOES-10. The thin line gives the total field, the thick line the north component. The shaded area indicates when the satellite was outside the magnetopause (except for fluctuations of the magnetopause after $2155 \mathrm{UT}$ ). (b) Calculated position of the magnetopause at GOES-10 location ( $R$, thick line) and in the solar direction $\left(R_{0}\right.$, thin line). (c) Same as (a), but for GOES8. (d) Same as (b), but for GOES-8. 

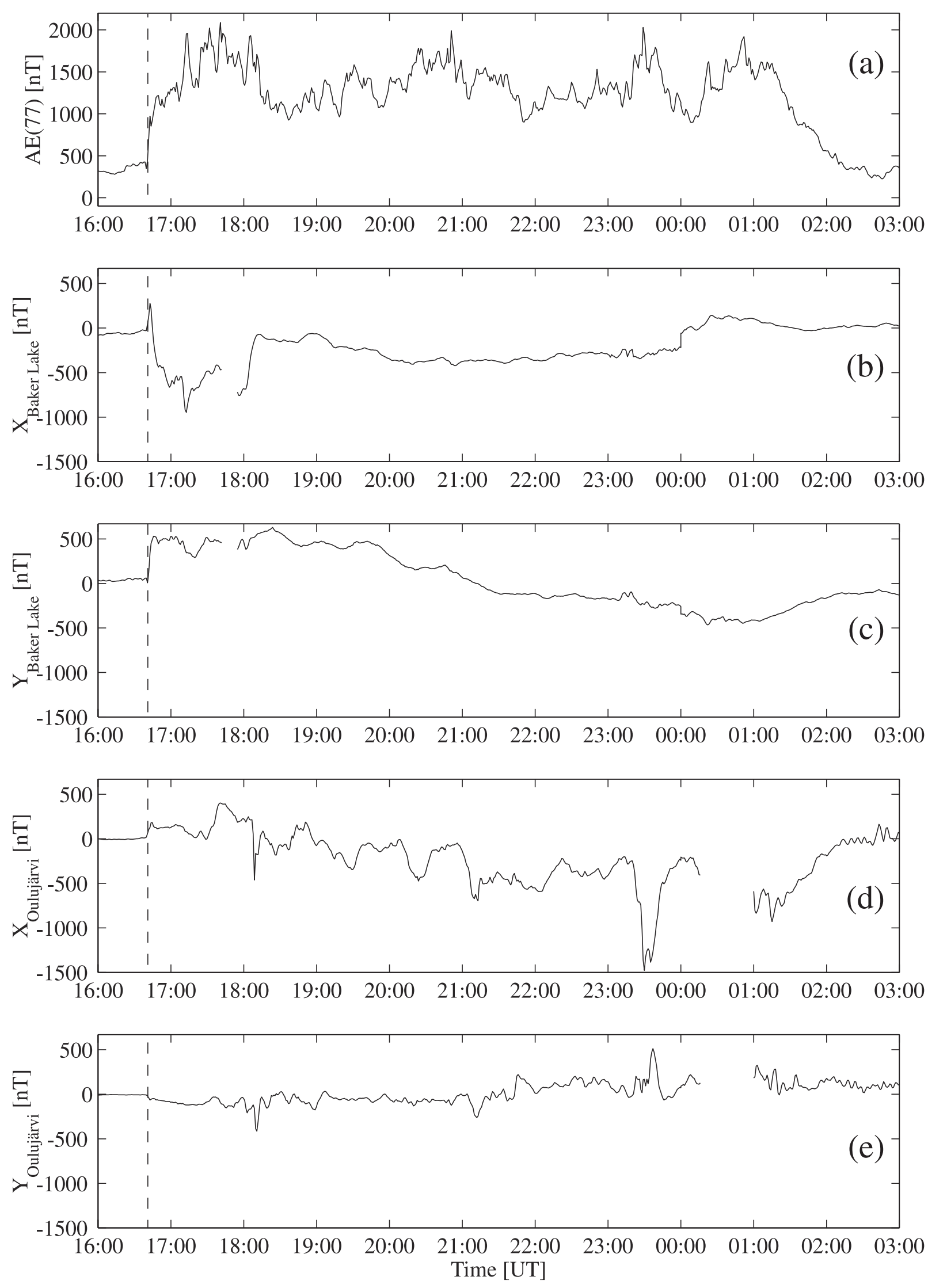

Figure 5. (a) AE index calculated using 77 magnetometer stations. (b-c) Magnetic $X$ and $Y$ components at Baker Lake. (d-e) Magnetic $X$ and $Y$ components at Oulujärvi. 


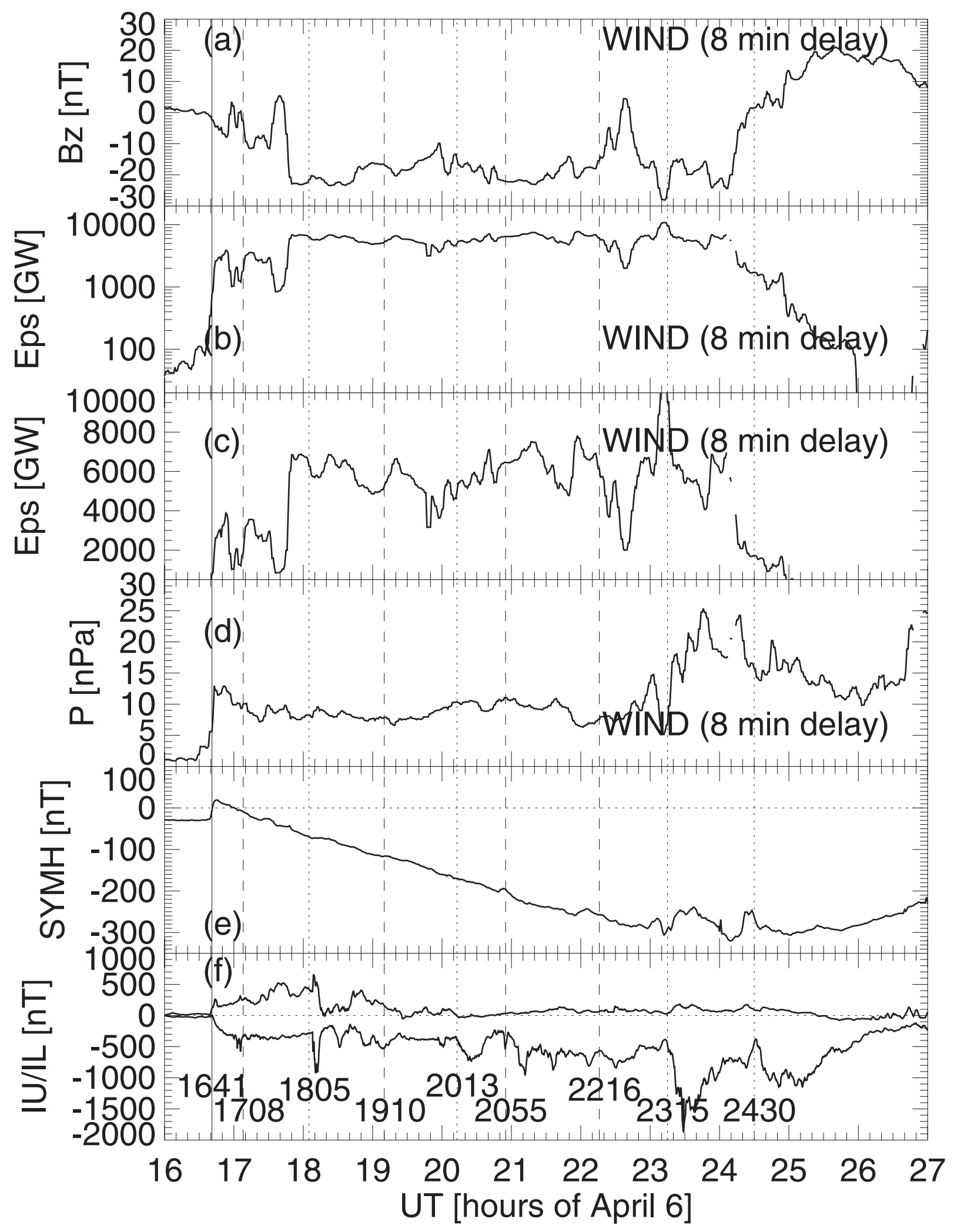

Figure 6. Interplanetary driver and magnetospheric activity on 6-7 April 2000. (a) IMF $B_{Z}$ from WIND shifted 8 min to the magnetopause. $(b-c) \epsilon$ from WIND shifted 8 min to the magnetopause. (d) Solar wind dynamic pressure shifted $8 \mathrm{~min}$ to the magnetopause. (e) SYM H index. (f) IU and IL indices created from the IMAGE magnetometer chain. The vertical solid line depicts the SSC time $1641 \mathrm{UT}$. The dotted lines show the electrojet activation times. 
the electrojet activity related to nightside magnetospheric processes. Eight electrojet activity onsets were identified from individual magnetometer data from around the globe (see discussion below); the onset times are indicated with the dotted lines. These eight activations and their times were chosen initially based on IMAGE magnetometer chain data. The aim was to pick independent "onsets" rather than "substorm intensifications." Later, magnetometer data from other chains were used to justify the onset identification, and the geosynchronous energetic particle injections were used to adjust the timings. These choices are, therefore, to some extent subjective, as no unique criteria for the "global onsets" could be identified. Consistent with the $\operatorname{AE}(77)$ shown in Figure 4, the electrojet activity enhanced directly after the shock arrival, and the maximum disturbances reached almost $2000 \mathrm{nT}$ around 2330 UT on 6 April. It is interesting to note that the SYM $\mathrm{H}$ index showed no response to the high-latitude activations before 2315 UT.

\subsubsection{Substorms and Convection Enhancements}

[30] In order to examine the electrojet enhancements in more detail, individual magnetometers from several stations around the Northern Hemisphere were examined. The IMAGE ground magnetic recordings from Fennoscandia are shown from 11 stations (ordered from north to south) in Figure $7 \mathrm{a}$. For tracking the global disturbances over the polar ionosphere, data from 10 magnetometers around the globe are shown in Figure $7 b$, where the geographic latitudes and longitudes of the recording stations are shown to the right of the image. The selected stations are a subset of the 77-station data set compiled to compute the AE index shown in Figure 5. The stations are ordered such that from top to bottom the stations range from noon to early morning hours to midnight to evening to early afternoon sector (see Figure 7c). Station names, acronyms, and locations are given in Table 1.

[31] Data from all 77 magnetometers were investigated by a spherical elementary current system analysis [Amm, 1997; Amm and Viljanen, 1999], which allows the ground magnetic data to be converted to ionospheric equivalent current density patterns (Figure 7c). The latitudinal spacing of the grid used for the elementary systems was $5^{\circ}$, the longitudinal spacing was $10^{\circ}$ and the total of 360 elementary systems were placed at an altitude of $100 \mathrm{~km}$. Stability of the solution to the underdetermined problem was obtained by using singular value decomposition. It should be noted that large spatial gaps in the data, especially over central Russia, result in unreliable estimates of the equivalent current patterns there. However, in the areas relatively densely covered by recording stations (Fennoscandia, Northern America, and Greenland), the estimates for the equivalent currents can be regarded as reliable. Figure $7 \mathrm{c}$ shows the current pattern at two time instants around the shock arrival, at 1641 and at 1643 UT. (Location of the magnetometer stations are also indicated in the figure.) The dayside currents were strongly enhanced shortly after the shock arrival at 1641 UT. At 1643 UT a strong westward current was developed near midnight as well. The global ionospheric current patterns are the subject of a separate study.

[32] Figure 8 shows data from the Los Alamos particle analyzers onboard three geostationary satellites, 1991-080, 1994-084, and 1989-046. The spacecraft were distributed in longitude such that at the storm onset, s/c 1994-084 was close to local midnight, whereas s/c 1991-080 was near midnight around $00 \mathrm{UT}$. Local noon and/or midnight are marked in the figures by arrows. At the storm onset, the lower energy electron fluxes increased at all three locations, whereas the highest energy channels in all locations showed a decrease of fluxes. This behavior is a characteristic response for an interplanetary shock arriving at the magnetopause. After the storm onset, the spacecraft near midnight recorded gradually decreasing values, with the first injection-like signatures at around 18 UT. Throughout the period, both electrons and protons (not shown) showed irregular pulses in the energy range below $1 \mathrm{MeV}$, typical of the stormtime injections [Reeves and Henderson, 2001]. The lack of clear (dispersionless) particle injections during the storm main phase makes it more difficult to use these data for substorm identification and timing. The flux dropout at s/c 1989-046 at 1816 UT indicates that the spacecraft moved across the magnetopause to the magnetosheath. This observation can be confirmed by analyzing the spectrograms utilizing the full energy range from $10 \mathrm{eV}$ to $10 \mathrm{MeV}$ (data not shown). This observation confirm the GOES observations of the magnetopause crossing, but further indicates that the magnetosphere was compressed even at local time 0715 far away from local noon in the morning sector.

[33] In the following analysis, we summarize the behavior of the eight activations that are marked with dotted lines in Figure 6. For each activation, we briefly describe the developing ionospheric current pattern and relate that to the geostationary measurements.

[34] The first activation was marked at 1708 UT. Close investigation of Figures 6 and $7 \mathrm{~b}$ shows that the disturbance was driven by the solar wind (southward turning of $B_{Z}$ and increase of the $\epsilon$ parameter), and that the disturbance propagated from the dayside stations toward the nightside. Furthermore, the spacecraft 1994-084 located close to midnight showed no signs of a particle injection near geostationary orbit. There was a proton signature at 1991-080 and 1984-084. The dispersion signatures indicate that this injection originated from very far in the dawn sector. Thus, rather than developing like a typical substorm with a nightside initiation, this activation seemed to be caused by enhanced convection, which in turn was driven by enhanced energy input to the system.

[35] The onset of the second activation at 1805 UT took place at low latitudes, with the strongest disturbance at the HAN station of the IMAGE magnetometer network at about $62^{\circ}$ geographic latitude. The onset was extremely sharp with time derivatives exceeding $5 \mathrm{nT} / \mathrm{s}$ at the southernmost IMAGE stations. On the other hand, the disturbance was relatively short-lived lasting only about $30 \mathrm{~min}$. This onset coincided with a clear injection at geostationary orbit near midnight, to the east of the IMAGE magnetometer chain.

[36] The activation marked at 1910 UT is difficult to time, as the activation started at the high-latitude (e.g., BJN at $74^{\circ}$ ) stations at $1850 \mathrm{UT}$ and the disturbance propagated equatorward to the auroral zone stations, which showed onset features at successively later times. At about 1910 UT, in a location conjugate to the magnetometers, geostationary electron fluxes showed a possible injection or a flux recovery. 


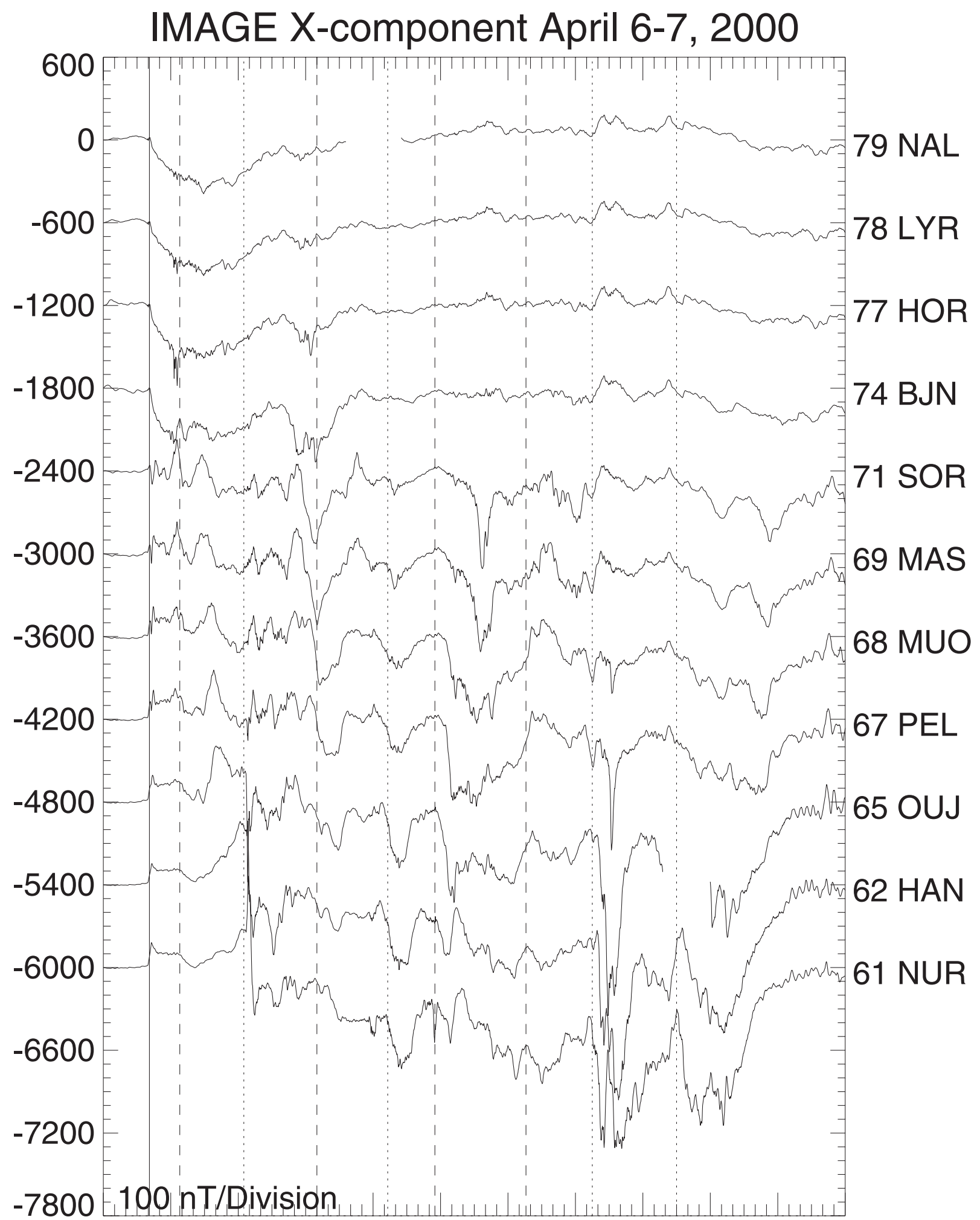

\section{$\begin{array}{llllllllllll}16 & 17 & 18 & 19 & 20 & 21 & 22 & 23 & 24 & 25 & 26 & 27\end{array}$ UT [hours of April 6]}

Figure 7a. $X$ components of 11 IMAGE magnetometer stations. Station acronyms and geographic latitudes are shown in the right. The vertical solid line depicts the SSC time 1641 UT. The dotted lines show the electrojet activation times. 
X-component April 6-7, 2000

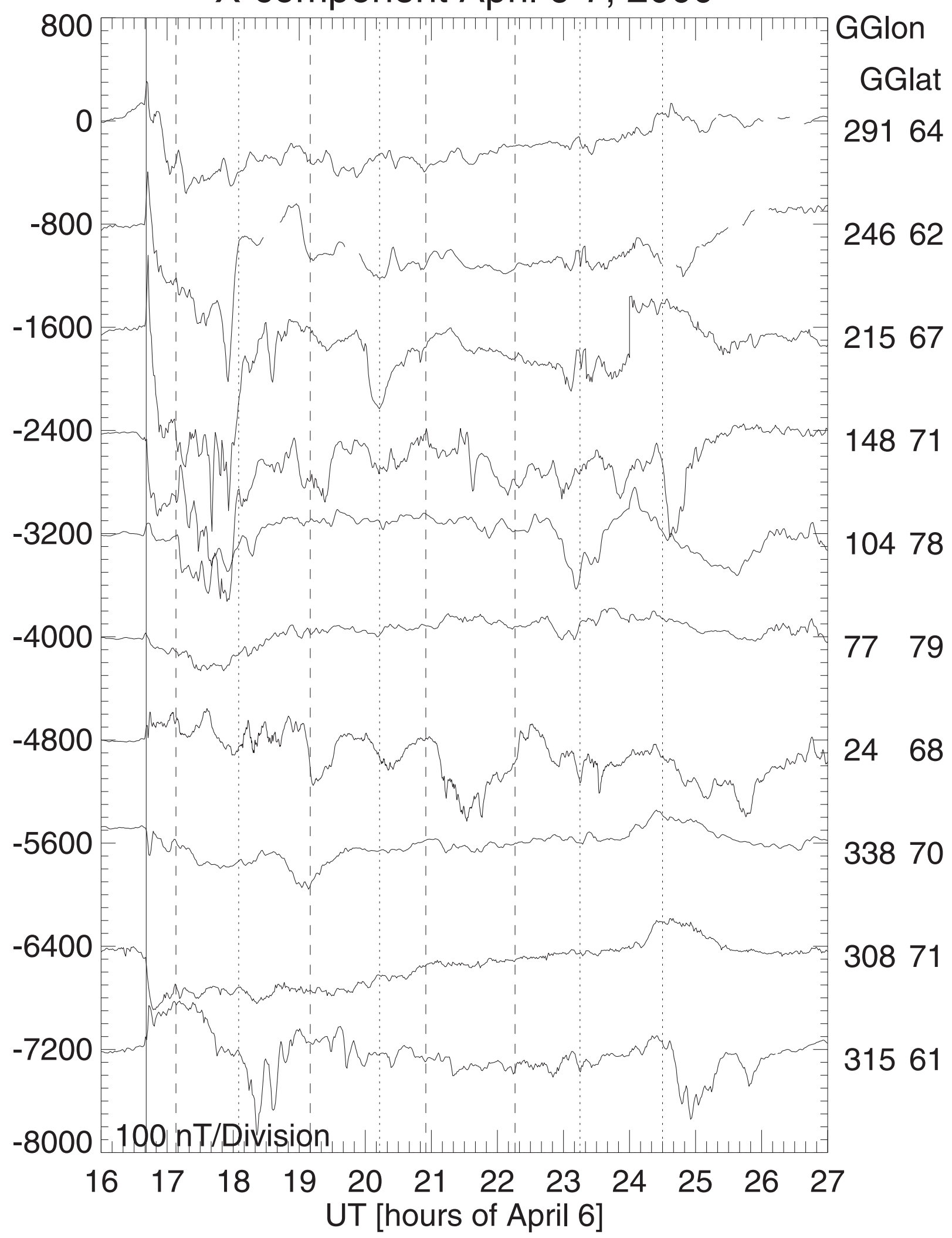

Figure 7b. $X$ components of 10 magnetometer stations around the world. Geographic latitudes and longitudes of the stations are shown in the right. The vertical solid line depicts the SSC time 1641 UT. The dotted lines show the electrojet activation times. 

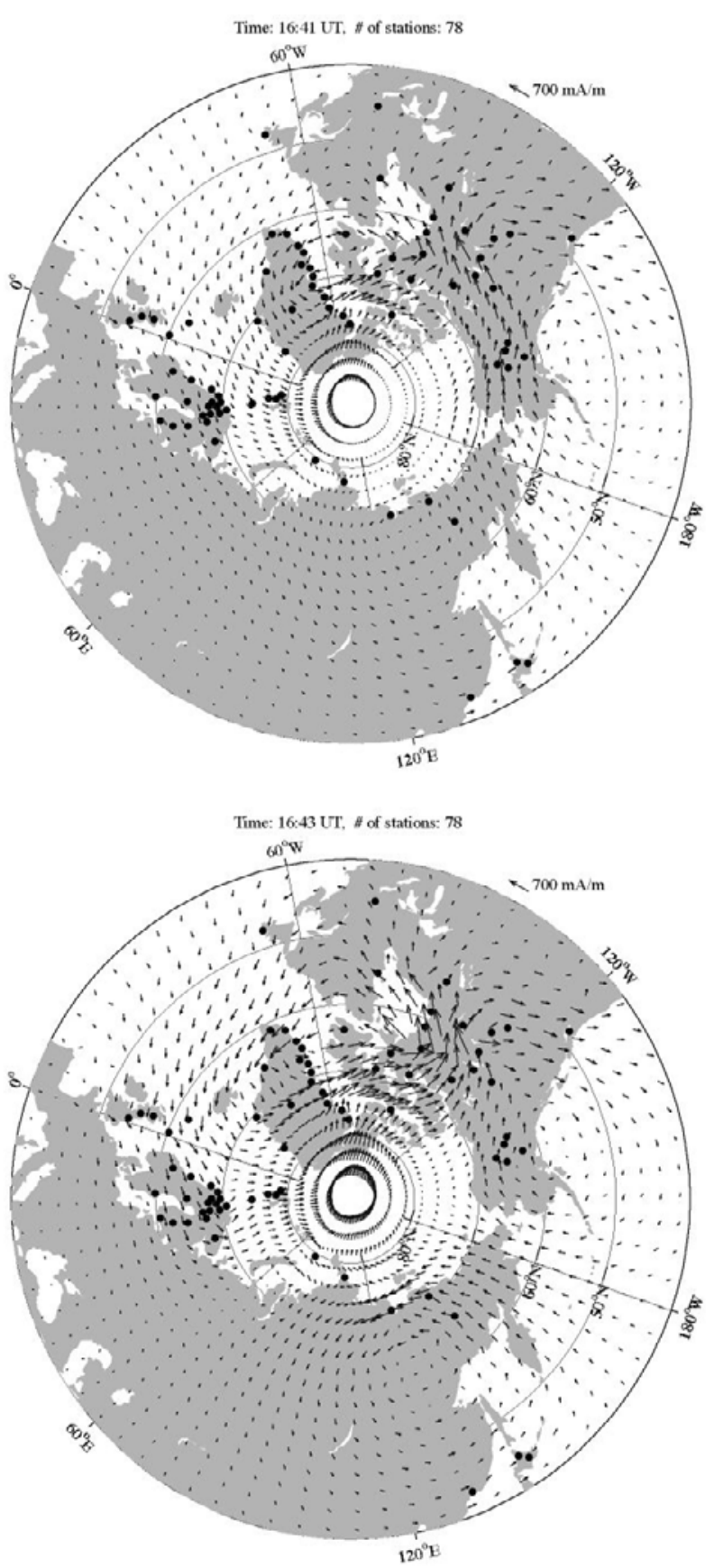

Figure 7c. Ionospheric equivalent current patterns at 1641 and 1643 UT calculated from observations at 78 magnetometer stations indicated by black dots. The maps are in geographic coordinates and the local noon is to the top.

[37] The onset at 2013 UT started at auroral latitudes with first signatures already at about 2000 UT at MUO and PEL at $67^{\circ}-68^{\circ}$ latitude. There was a coincident small injection or flux recovery at geostationary orbit. The onset at 2055 UT was initiated from low latitudes (NUR-OUJ, $61^{\circ}-65^{\circ}$ latitude). This event was characterized by sharp gradients at the southernmost IMAGE station NUR. There were no injection signatures at geostationary orbit. The activation at 2216 UT started from the latitudes $61^{\circ}-62^{\circ}$ and propagated poleward, and was also not associated with injection signatures at geostationary orbit.

[38] Finally, two large onsets took place at 2315 UT and 0030 UT. The 2315 UT onset initiated from the southern stations (NUR, $61^{\circ}$ ), and was associated with very large gradients in the local magnetic field. This activation was coincident with a short-lived injection near local midnight. The solar wind and IMF data show a deep minimum of the IMF Z-component followed by strongly oscillating and enhancing solar wind pressure. This onset was thus likely associated with the strongly enhanced energy input and the dynamic variations in the magnetosphere caused by the oscillating plasma pressure. The last onset at 0030 UT initiated from NUR $\left(61^{\circ}\right)$, and followed the final northward turning of the IMF.

[39] In summary, out of eight activations, four showed substorm-like behavior $(1805,2013,2315$, and 0030 UT, expansion from an initiation region mainly poleward and westward, electrojet enhancement associated with injection signatures at geostationary orbit). Four activations showed quite different behavior (1708, 1910, 2055, and 2216 UT), with typically activation spreading either from the west eastward and equatorward or from the north equatorward. These activations were not associated with clear injection signatures at the nightside geostationary orbit. Therefore, our interpretation is that their morphology is quite distinct from the classical substorm sequence [Akasofu, 1964; Baker et al., 1996].

[40] In order to illustrate the behavior atypical for substorms, we discuss two of the nonsubstorm activations (the 1708 UT initial onset and the 1910 UT activation) and one substorm onset (1805 UT) in more detail. Figure 9 shows selected stations from the IMAGE magnetometer chain and data from two LANL particle analyzers onboard two geostationary spacecraft. As pointed out above, spacecraft 1991-084 was located to the east of the IMAGE magnetometer chain, while spacecraft 1991-080 was located to the west of the IMAGE chain. Thus, either of the spacecraft should be able to record substorm onset-associated electron flux enhancements, if the onset was visible in the IMAGE magnetometers.

[41] The top panel shows data from five IMAGE stations, ordered from north to south, ranging from $74^{\circ}$ to $60^{\circ}$ magnetic latitude. The solid gray lines indicate the equatorward expansion of the events 1708 UT and 1910 UT. Furthermore, these two events show no clear associated changes in the geosynchronous particle fluxes. On the other

Table 1. Names, Acronyms, and Geographic Coordinates of the Magnetic Stations Whose Data are Shown in Figure 7b

\begin{tabular}{lccc}
\hline \multicolumn{1}{c}{ Name } & Acronym & Geogr. lat. & Geogr. long. \\
\hline Narsarquad & NAQ & $61.16^{\circ}$ & $314.56^{\circ}$ \\
Uummannaq & UMQ & $70.68^{\circ}$ & $307.87^{\circ}$ \\
Ittoqqortoormiit & SCO & $70.48^{\circ}$ & $338.03^{\circ}$ \\
Cape Chelyuskin & CCS & $77.71^{\circ}$ & $104.27^{\circ}$ \\
Vyze Island & VYZ & $79.48^{\circ}$ & $75.98^{\circ}$ \\
Chokurdakh & CHD & $70.62^{\circ}$ & $147.89^{\circ}$ \\
Zyryanka & ZYK & $65.75^{\circ}$ & $150.78^{\circ}$ \\
Fort Yukon & FYK & $66.56^{\circ}$ & $214.78^{\circ}$ \\
Yellowknife & YKL & $62.48^{\circ}$ & $245.52^{\circ}$ \\
Iqaluit & IQA & $63.75^{\circ}$ & $291.48^{\circ}$ \\
\hline
\end{tabular}




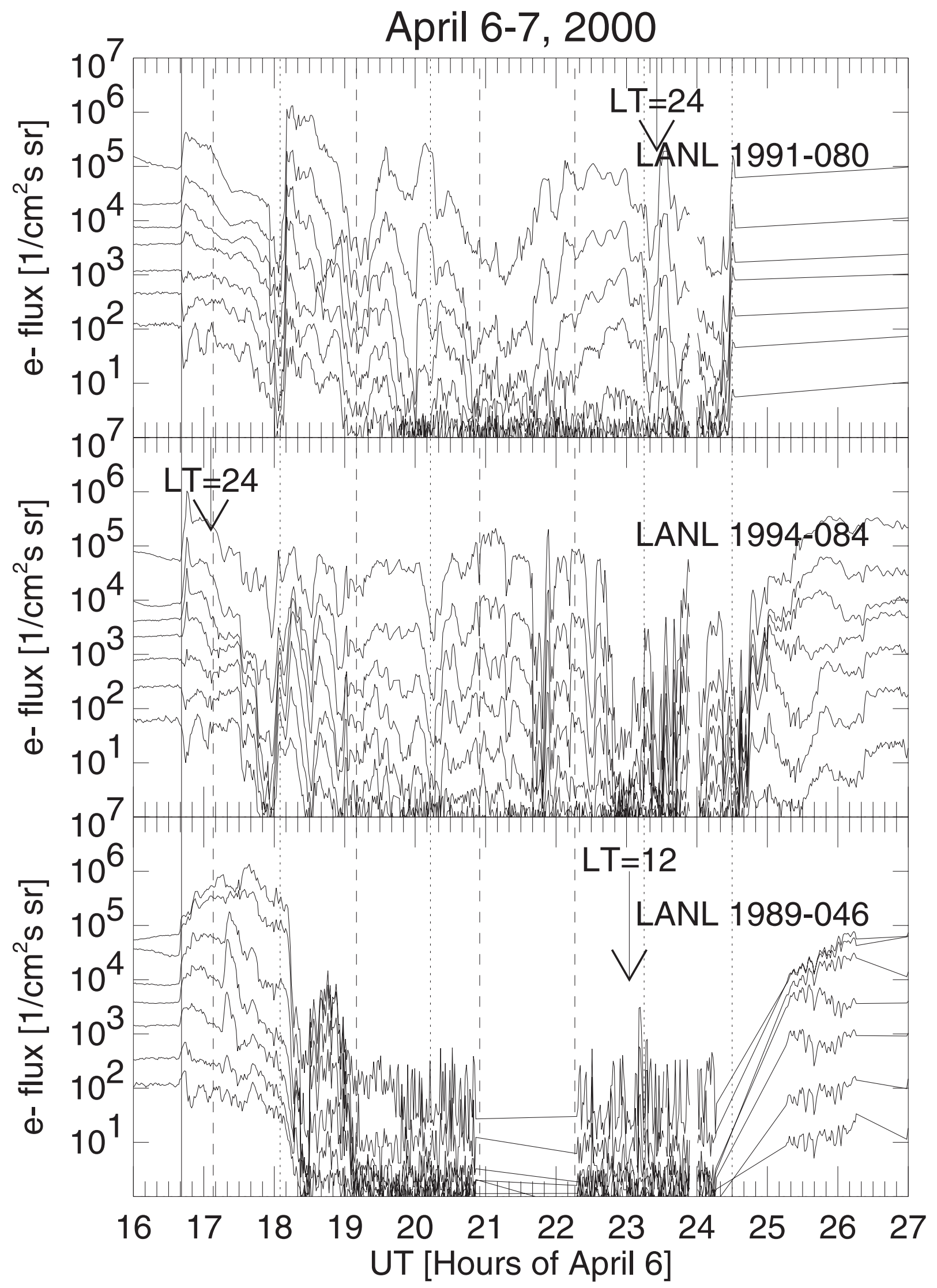

Figure 8. Differential electron fluxes from (top) s/c 1991-080, (middle) s/c 1994-084, and (bottom) s/c 1989-046. The energy channels shown are from top to bottom: $50-75,75-105,105-150,150-225$, $225-315,315-500$, and 500-750 keV. 


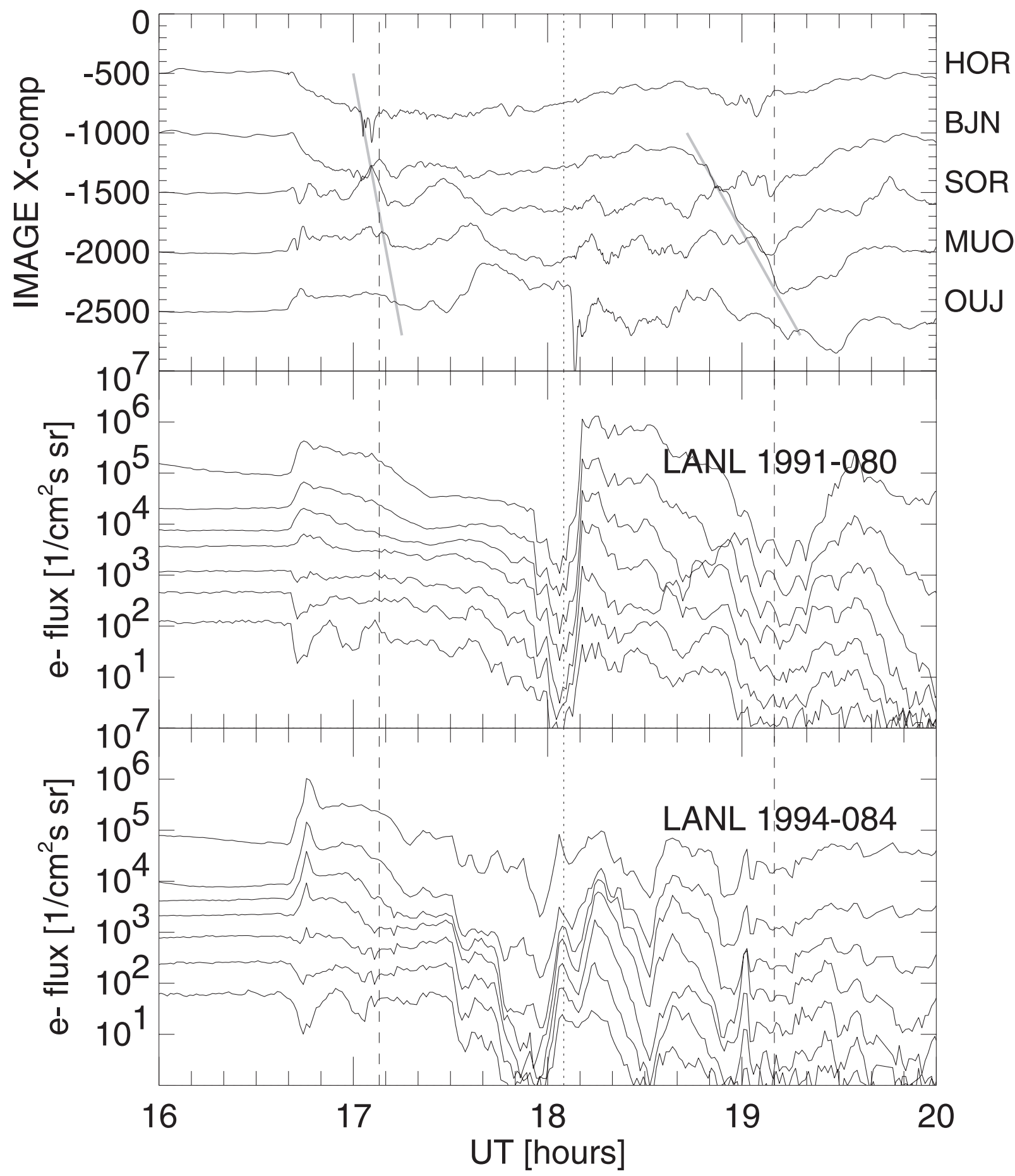

Figure 9. Five selected stations from Image magnetometer chain ordered from north to south (top). The grey lines indicate the expansion of the activations 1708 and 1910 UT. Two bottom panel show differential electron fluxes from s/c 1991-080 and s/c 1991-084.

hand, the 1805 UT event is associated with an electron injection, which is visible first at s/c 1994-084, and a few minutes later at 1991-080. Both injections are dispersionless. This indicates that the injection front is wide enough to engulf both spacecraft, and that the injection front expanded from the tail earthward.

[42] Strong westward electrojet enhancement associated with an earthward propagating injection front is a typical substorm onset sequence, as demonstrated in numerous earlier studies [e.g., Baker et al., 1996, and references therein]. Therefore, despite the ongoing storm activity, the 1805 UT onset seems to have the major characteristics of a substorm onset. On the other hand, the two other electrojet enhancement clearly show propagation from the poleward edge of the auroral oval toward lower latitudes. If one assumes that the substorm current system is associated with 
a disruption of the tail current within the central plasma sheet, the observed equatorward propagations during the 1708 and 1910 UT enhancements are not consistent with substorm evolution. Furthermore, neither of the onsets show signatures of electron injection, either westward or eastward of the magnetometer chain. This further demonstrates a lack of current disruption and major magnetospheric reconfiguration that would be expected for a substorm onset. Therefore, even if in magnetic indices these disturbances can look quite similar to substorms, their dynamic evolution is quite different.

[43] Some of the activations (1708, 2216, 2315, and 0030 UT) could clearly be associated with changes in the solar wind and IMF parameters either southward or northward turning, and thus their timing was controlled by these solar wind variations. On the other hand, the onsets at 1805, 2013, and 2055 UT seemed to occur without an apparent solar wind trigger, and thus occurred as a consequence of magnetospheric dynamics. Note that the onset at 1910 UT was associated with small increase in epsilon due to slight decrease of IMF Z-component but it is unclear if this can be considered as an external control at the onset. The onsets moved toward lower latitudes as the storm progressed such that the last four onsets all initiated well equatorward of the typical auroral oval region. This is of course a consequence of the storm-associated equatorward motion of the auroral oval and electrojet pattern. The strong solar wind pressure throughout the main phase, which pushed the magnetopause inside or close to the geostationary orbit throughout this period, enhanced the dynamic coupling with the solar wind and the magnetosphere. However, even under such circumstances the magnetosphere produced activations independent of the solar wind variability.

[44] Two increases in the SYM H index were observed at 2315 and 0015 UT. The first coincided with a substorm onset, but also with a rapid change in the solar wind driver properties. The second increase preceded the substorm onset, but was coincident with an IMF northward turning. Thus, in this event the substorm activity did not seem to lead to marked changes in the midlatitude magnetic recordings; rather the SYM H responded directly to the driving solar wind and IMF properties.

\subsection{Geomagnetically Induced Currents}

[45] The induction phenomena driven by ionospheric current systems are the ground end of geomagnetic storms. The electric field produced by the rapidly varying magnetic field induces an electric field inside the Earth, which drives currents in long man-made conductor systems, causing harm to power systems and buried pipeline networks [e.g., Boteler et al., 1998]. The geomagnetically induced currents (GIC) flowing in the Finnish natural gas pipeline network have been measured since November 1998 [Pulkkinen et al., 2001] in Mäntsälä (Figure 10a) at the center of the network. The measurements are realized by placing a magnetometer above the pipeline, which then measures the Biot-Savart field produced by the current flowing along the pipeline.

[46] The GIC flowing in the Finnish pipeline during 67 April 2000, is shown in Figure 10b together with the time derivative of the north component of the ground magnetic field $(d X / d t)$ as measured at the nearby NUR

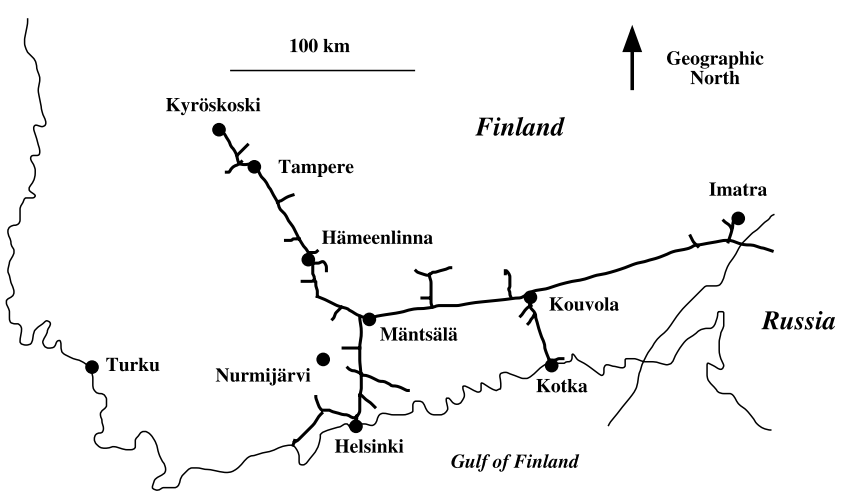

Figure 10a. Map showing the Finnish natural gas pipeline network at geographic latitudes $60-63^{\circ}$.

station. Positive direction of the GIC is chosen to be eastward. As demonstrated in Figure 10b, there is good correlation between the GICs and $d X / d t$. In general, the magnitude of the time derivative of the geomagnetic field is a good indicator of the GIC intensity [Viljanen et al., 2001].

[47] At the storm onset, the sudden impulse caused a pulse-shaped structure in the ground magnetic field. The pulse produced a time derivative of about $1.3 \mathrm{nT} / \mathrm{s}$ at NUR, which was associated with a 3.5 A GIC at Mäntsälä, with GICs over $1 \mathrm{~A}$ recorded over a $5 \mathrm{~min}$ period.

[48] At 1807 UT, following the substorm onset, a very intense GIC event took place. Both polarities of $d X / d t$ and GIC were observed, with peak-to-peak amplitudes of $15 \mathrm{nT} /$ s or $34 \mathrm{~A}$, respectively. GICs over $1 \mathrm{~A}$ were observed for about 7 min. During the time period 1950-2200 UT, several intense GIC spikes were recorded. The 2055 UT spike was associated with an extremely localized disturbance at NUR, which occurred coincident with one of the activations without clear substorm onset signatures discussed above.

[49] At 2305 UT, Mäntsälä was under the enhanced westward electrojet region. Changes in the electrojet amplitude $(d X / d t \simeq-2 \mathrm{nT} / \mathrm{s})$ caused a smooth westward GIC of -5 A prior to the substorm onset at $2315 \mathrm{UT}$. The peak values of $d X / d t$ and GIC at $2325 \mathrm{UT}$, were $5 \mathrm{nT} / \mathrm{s}$ and $23 \mathrm{~A}$. GICs over 5 A were observed for a period of about $10 \mathrm{~min}$, and intensifications and weakenings of the substorm electrojet caused the GIC to oscillate rapidly throughout the substorm. During the substorm recovery phase, rapid oscillations of the GICs having amplitudes of several amperes were observed.

[50] At the end of the storm main phase, 0000-0130 UT on 7 April, several intense $d X / d t$ and GIC values were observed, the largest ones being $-14 \mathrm{~A}$ and $-3.5 \mathrm{nT} / \mathrm{s}$, associated with the changes in the westward electrojet. During the storm recovery phase (0200-0430 UT) magnetic pulsations in the Pc5 range (150-600 s) caused GIC activity with amplitudes of several amperes.

[51] The three most intense GIC events were all associated with activations, at 1805, 2055, and 2315 UT. However, there were no common characteristics in the substorm behavior that could be associated with these events: The 1805 UT substorm was driven by internal magnetospheric processes. 
GIC at Mäntsälä on April 6-7, 2000

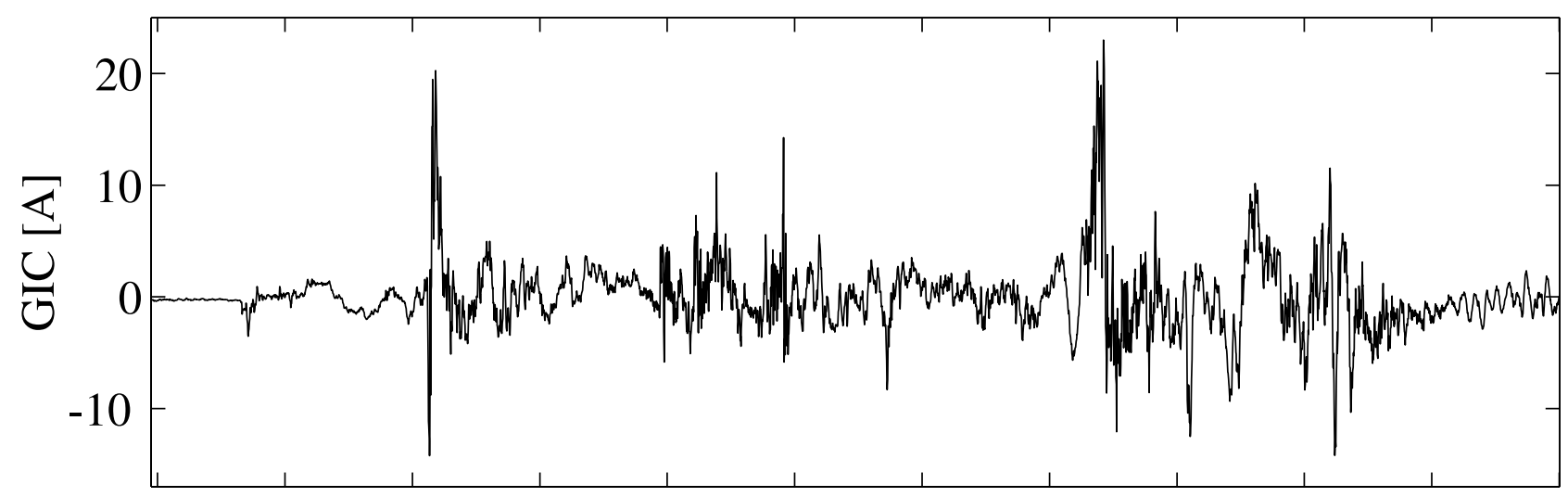

$\begin{array}{llllllllllllll}16: 00 & 17: 00 & 18: 00 & 19: 00 & 20: 00 & 21: 00 & 22: 00 & 23: 00 & 00: 00 & 01: 00 & 02: 00 & 03: 00\end{array}$

\section{Time derivative of $\mathrm{X}$ at Nurmijärvi}

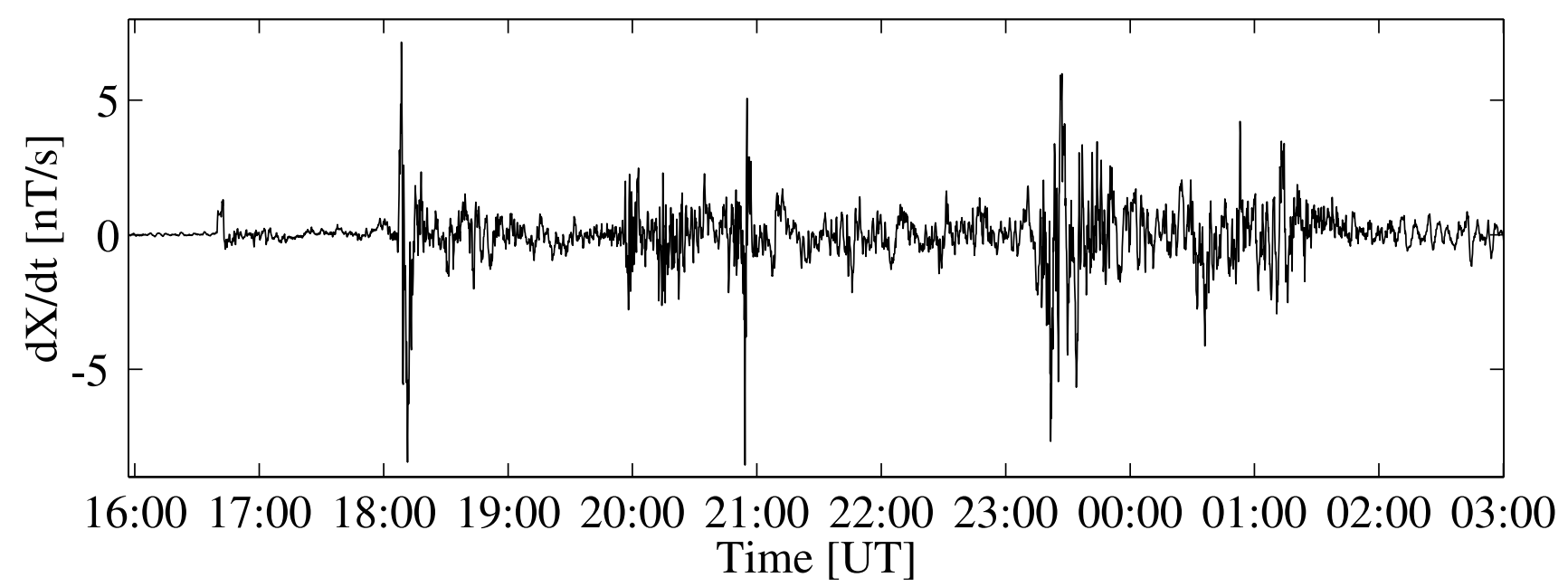

Figure 10b. (top) Geomagnetically induced current measured in Mäntsälä, Finland, and (bottom) time derivative of the north component of magnetic field at the closeby station Nurmijärvi.

The 2055 UT activation was not a typical substorm onset, and the entire GIC event was very localized. On the other hand, the 2315 UT onset was closely associated with the pulsating solar wind pressure just behind a strongly negative IMF $B_{Z}$. The processes that led to these extreme temporal magnetic field gradients and hence the GIC events will be investigated further in a separate paper.

\section{Discussion}

[52] The detection of a full halo CME and accompanying solar activity near the center of the solar disc raises attention among space weather forecasters. Despite the western location of the halo CME detected by LASCO on 4 April 2000, and the associated solar activity (filament disappearance and C-class flare), the largest magnetic storm of the year 2000 took place 2 days later. In this paper we have made a thorough analysis of this storm using observations from the solar wind upstream of the Earth, dayside and nightside magnetosphere, auroral ionosphere as well as of the geomagnetically induced currents.
[53] A strong interplanetary shock was detected at 1 AU on 6 April by several spacecraft monitoring the solar wind. After the shock passage, the spacecraft encountered the sheath region plasma. This storm was caused by the very strong sheath region, as at the time of the arrival of ICME signatures around 06 UT on 7 April the storm was already in the recovery phase. After the start of the ICME signatures the magnetic field intensity decreased within a couple of hours to very low values (about $5 \mathrm{nT}$ ) suggesting that spacecraft intercepted the ICME far from its center. Various mechanisms that can cause intense southward (or northward) magnetic field values in the CME sheath region have been suggested [Gosling and McComas, 1987; Tsurutani and Gonzalez, 1997], and Tsurutani et al. [1988] have shown that the sheath regions alone can drive intense (Dst $<-100 \mathrm{nT}$ ) magnetic storms.

[54] Many observations described in sections 3.1, 3.2, and 3.3 suggest that the spacecraft and the Earth hit only the flanks of the ICME. Most of the CME material in coronagraph images and the associated activity, the flare (N18W58) and the filament disappearance (N25W58) occurred near the western limb of the Sun. Solar wind measurements also 
support this scenario as described above. Solar wind signatures characteristic to magnetic clouds [Zwickl et al., 1983; Gosling, 1990; Henke et al., 1998] (low beta and temperature, enhanced $\mathrm{O}^{+7} / \mathrm{O}^{+6}$ and $n_{\mathrm{\alpha}} / n_{p}$ ratio, counterstreaming suprathermal electrons) and the association to the filament disappearance suggest that the launched CME had magnetic cloud topology. As spacecraft intercepted only flanks of this magnetic cloud we could not calculate its orientation from magnetic field data. But as the associated filament was vertically elongated along the surface of the Sun we can infer that the magnetic cloud likely was highly inclined relative to ecliptic plane [Marubashi, 1997]. The obtained shock normal had large latitudinal angle, $\theta \simeq-40^{\circ}$ consistent with source in the Northern Hemisphere and the longitudinal angle $\phi \simeq 170^{\circ}$ that is consistent with the CME originating from west but the deviation from $180^{\circ}$ is too small to be consistent with the flare and filament location near the western limb. However, as we discussed in section 3.2 the determination of the shock normal for this event was difficult and above mentioned values should be considered only as rough estimates. Note, that as the shock front is not planar but curved around the ejecta, the axis of the magnetic cloud should have had positive inclination relative to the ecliptic to explain the large negative latitudinal angle of the shock normal. Finally, the magnetosphere compression was stronger at the dawnside than at the duskside. In the case of a magnetic cloud having its axis lying in the ecliptic plane and passing the Earth's magnetosphere mostly dawnside we would expect a much larger longitudinal angle and smaller latitudinal angle. Thus, a magnetic cloud having high inclination is more consistent with the obtained shock normal orientation than a magnetic cloud lying in the ecliptic plane. Summarizing all observations we suggest a scenario where the magnetic cloud expanded from the Sun to the interplanetary space somewhat away from the Sun-Earth line with axis having high positive inclination with respect to the ecliptic plane. This magnetic cloud swept the Earth's magnetosphere from above and dawnside so that the measured ICME signatures exhibit lower edge of this larger scale structure. As there is still lot of uncertainties concerning the 3D structure of the ejecta and the data are limited it would not be correct to speculate any further.

[55] Besides CMEs, corotating interaction regions (CIR) [Smith and Wolf, 1976] sometimes drive intense magnetic storms. However, we do not believe that this solar wind event was a CIR as the observed shock on 6 April was strong and CIRs are fully developed (with forward and reverse shocks) only beyond the Earth orbit. Also the magnetic field direction within the developing CIRs fluctuates rapidly so they do not usually cause very intense magnetic storms [Gosling et al., 1991].

[56] Usually, the long-lasting negative $B_{Z}$ events are associated with ICMEs, particularly with magnetic clouds during which the solar wind density and dynamic pressure are typically low. This event supports the view that the high dynamic pressure enhances the ring current injection through solar wind density driving of the "superdense" plasma sheet [Borovsky et al., 1997]. At the same time it is not in contradiction with the results by O'Brien and McPherron [2000], who claimed that the pressure does not have an independent role, as in this case the high plasma pressure was associated with long-lasting southward $B_{Z}$. While Huttunen et al. [2002] showed that usually sheath regions cause relatively stronger $\mathrm{Kp}$ values than Dst values, in this event the negative magnetic field values were sustained exceptionally long for the sheath region, and thus both Dst and Kp were greatly enhanced.

[57] The geomagnetic activity was examined in detail using ground magnetometer data from 77 stations around the Northern Hemisphere polar, auroral, and midlatitude regions. Individual electrojet enhancements were identified and analyzed in the context of the generally accepted scheme of substorm evolution [e.g., Baker et al., 1996, and references therein]: In the ionosphere, the substorm onset is characterized by the onset of a strong westward electrojet current with associated field-aligned currents forming the substorm current wedge. The expansion of the magnetic disturbance (as well as the associated auroral bulge) is mostly poleward and westward. In the magnetotail, at or near substorm onset time, the inner magnetotail field becomes more dipolar, the energetic particle fluxes increase rapidly, and in the midtail both earthward and tailward fast plasma flows are observed. These signatures are independent of the assumed substorm trigger mechanism. For this study, ionospheric data of the electrojet dynamics as well as inner magnetosphere energetic particle records were used to examine the substorm evolution. Unfortunately, no magnetotail data of the fast flows are available for this event.

[58] Using these criteria, only four out of eight clear electrojet enhancements could be categorized as substorms, even if we allow for missing signatures due to possible lack of observations at the exact site of localized disturbance. In most of these nonsubstorm cases, the magnetic disturbance was observed to begin from high latitudes and/or away from the midnight sector, and the predominant travel direction was toward midnight and equatorward. Furthermore, these activations did not seem to be associated with configuration changes in the inner magnetotail (either particle injections or magnetic field dipolarizations, both of which can be observed as flux-level changes in energetic particle observations). Therefore, we assume that these activations were direct responses to the solar wind and/or IMF driving, and were not associated with dynamic configuration changes in the magnetotail.

[59] Furthermore, several of the more typical substorm onsets, especially the last two large substorms, were associated with clear changes in the solar wind and IMF configuration, thus suggesting that they were driven by the external driver [Lyons, 1996]. Thus, it seems that during storms ionospheric (and possibly also magnetospheric) dynamics is quite directly controlled by the solar wind and IMF variations, with only occasional situations where the internal dynamics can evolve to a state where a more "typical" substorm evolution (tail instability leading to substorm current wedge currents and auroral bulge development) can take place.

[60] In addition to the major "onsets" discussed in detail here, there was significant dynamic behavior observed as well in the ground-based magnetometer data, in geosynchronous particle fluxes, as in other observations. These activations were not large enough or sufficiently coherent to be included in the onset list, but indicate the nearly 
continuous forcing of the solar wind on magnetospheric dynamics. Some of these nonsubstorm activations most probably share the properties of equatorward driving arcs, $\mathrm{N}$-S-aligned auroral arcs, auroral streamers or poleward boundary intensifications as discussed by Henderson et al. [1998], Sergeev et al. [1999], and Lyons et al. [1999]. These studies discuss the magnetospheric and ionospheric signatures associated with these auroral activations. They conclude that while many of the physical processes involved are the same as during substorms, the manifestations are quite different because of the strong and continued solar wind driving.

[61] This large magnetic storm caused the second largest GIC measured at Mäntsälä since the beginning of continuous observations in November 1998. So far, the largest GIC (32 A) was measured on 11 November 2001. According to statistics based on magnetic data over several solar cycles and model calculations by Pulkkinen et al. [2001], GICs over 25 A can be expected once per year at the Mäntsälä site. Thus, although not being extreme, the April 2000 storm was a quite intense GIC event also in a statistical sense.

[62] Intense GICs investigated in this study imply that a large portion of the ionospheric drivers of the large GIC are related to substorm activity. This makes perfect sense since substorms are capable of producing large temporal variations in ionospheric current systems, which then induce that intense ground electromagnetic fields. However, no common characteristics in the substorm behavior, that could be associated with these events, were found. More importantly, the detailed behavior of the ionospheric currents during these events was different from case to case. This suggests that both temporal and spatial structures of the geoelectric field driving the GICs are highly variable. From the GIC forecasting point of view, all this means that if the time and place of the substorm-related ionospheric current variations can be predicted, also the time and the location of most GIC enhancements can be predicted. In order to compute the detailed distribution of GIC in a specific technological system, one needs to know the detailed structure of the induced geoelectric field. In light of this study, it is quite obvious that requirements for a good GIC forecasting model, capable of producing such detailed structures, are extremely demanding.

[63] In this strongly driven case, it is interesting to note that the Dst (as well as the high-resolution SYM-H index) evolution is completely decoupled from the high-latitude magnetic variations. The original concept of a substorm was that it is a smaller activation of which storms were composed [Chapman, 1962]. It has been argued that the energetic particle injections at substorm onset provide the population necessary for the stormtime ring current formation, and hence the substorm activity is an essential component for the storm evolution [Davis and Sugiura, 1966]. However, this view has been contradicted by several studies. For example, linear filter analyses suggest that Dst has high correlation with the driver parameters, whereas its correlation with the AE indices (indicating substorm activity) is lower [for a review, see McPherron, 1997]. As the SYM-H index seems uncorrelated with the high-latitude magnetic activity, this would lend support to the conclusion that substorms do not play an important role in the storm evolution. Furthermore, as many of the high-latitude acti- vations in the $\mathrm{AE}$ index are not substorms that would be associated with injections at geostationary orbit, these data would suggest that the substorm-associated energetic particle injections are not the primary cause of the intense ring current formation.

[64] The high dynamic pressure throughout the storm makes this event quite exceptional. Therefore, more storms need to be analyzed before the conclusions about the stormtime substorms or nonsubstorms and their role in the ring current evaluation can be generalized. However, this study alone demonstrates that under high dynamic pressure and strongly southward interplanetary field, the magnetotail can assume a much more driven state and possibly quite different dynamical behavior than during the loadingunloading sequence of isolated substorms. Furthermore, under such circumstances, most of the ionospheric electrojet activity is directly controlled by the IMF and solar wind variations and do not seem to couple to dynamic magnetotail processes.

[65] Acknowledgments. We thank R. Lepping for the WIND magnetic field data and A. Lazarus for the WIND solar wind data. We also thank C. W. Smith for the ACE magnetic field data and D. J. McComas for the ACE solar wind data. These data were obtained through Coordinated Data Analysis Web (CDAWeb). We thank the EIT, LASCO, and CELIAS teams of SOHO for the Solar and Solar wind observation. SOHO is a mission of international cooperation between ESA and NASA. The CME catalog used is generated and maintained by the Center for Solar Physics and Space Weather, The Catholic University of America, in cooperation with the Naval Research Laboratory and NASA. Other solar information was obtained from the Space Environment Center, Boulder, CO, NOAA, U.S. Department of Commerce. The Kp and Dst indices were obtained from the World Data Center C2 in Kyoto.

[66] The authors acknowledge the work made at the numerous institutions involved in the construction, maintenance, and operation of the magnetic observatories whose data have been used in the analysis: M. Engebretson at Augsburg College and W. J. Hughes at Boston University (MACCS), J. Olson and Debi-Lee Wilkinson at Geophysical Institute of the University of Alaska (GIMA), F. Creutzberg at Canadian Space Agency and J. Samson at University of Alberta (CANOPUS), K. Yumoto at Kyushu University (210 Magnetic Meridian Chain), I. R. Mann and D. K. Milling at University of York (SAMNET), J. Watermann at Danish Meteorological Institute and C. R. Clauer at University of Michigan (Greenland magnetometers), L. Newitt at Geological Survey of Canada (GSC magnetometers), Oleg Troshichev at Arctic and Antarctic Research Institute (Russian magnetometers), and A. Viljanen at Finnish Meteorological Institute (IMAGE). The work of Emilia Huttunen was supported by the ANTARES program, Minna Palmroth by the MaDaMe, and Antti Pulkkinen by the project 47680 of the Academy of Finland.

\section{References}

Akasofu, S.-I., The development of the auroral substorm, Planet. Space Sci., 12, 273-282, 1964.

Akasofu, S.-I., Interplanetary energy flux associated with magnetospheric substorms, Planet. Space Sci., 27, 425-431, 1979.

Akasofu, S.-I., Relationship between AE and Dst indices during geomagnetic storms, J. Geophys. Res., 86, 4820, 1981.

Amm, O., Ionospheric elementary current systems in spherical coordinates and their application, J. Geomagn. Geoelectr., 49, 947, 1997.

Amm, O., and A. Viljanen, Ionospheric disturbance magnetic field continuation from the ground to ionosphere using spherical elementary current systems, Earth Planets Space, 51, 431, 1999.

Baker, D. N., T. I. Pulkkinen, V. Angelopoulos, W. Baumjohann, and R. L. McPherron, Neutral line model of substorms: Past results and present view, J. Geophys. Res., 101, 12,975-13,010, 1996.

Borovsky, J. E., M. F. Thomsen, and D. J. McComas, The superdense plasma sheet: Plasmapheric origin, solar wind origin, or ionospheric origin?, J. Geophys. Res., 102, 22,089, 1997.

Borrini, G., J. T. Gosling, S. J. Bame, and W. C. Feldman, Helium abundance enhancements in the solar wind, J. Geophys. Res., 87, 7370-7378, 1982.

Boteler, D., R. J. Pirjola, and H. Nevanlinna, The effects of geomagnetic disturbances on electrical systems at the Earth's surface, Adv. Space Res., 22, 17, 1998 . 
Bothmer, V., and R. Schwenn, Eruptive prominences as sources of magnetic clouds in the solar wind, Space Sci. Rev., 70, 215-220, 1994.

Brueckner, G. E., et al., The large angle spectroscopic coronagraph (LASCO), Sol. Phys., 162, 357-402, 1995.

Burlaga, L. F., Magnetic clouds and force-free fields with constant alpha, J. Geophys. Res., 93, 7217-7224, 1988

Burlaga, L., E. Sittler, F. Mariani, and R. Schwenn, Magnetic loop behind an interplanetary shock: Voyager, Helios, and IMP 8 observations, J. Geophys. Res., 86, 6673-6684, 1981.

Burton, R. K., R. L. McPherron, and C. T. Russell, An empirical relationship between interplanetary conditions and Dst, J. Geophys. Res., 80, 4204, 1975 .

Chapman, S., Earth storms: Retrospect and prospect, J. Phys. Soc. Jpn., 17(Suppl. A-1), 6-16, 1962.

Davis, T. N., and M. Sugiura, Auroral electrojet activity index AE and its universal time variations, J. Geophys. Res., 71, 785-801, 1966.

Gonzalez, W. D., and B. T. Tsurutani, Criteria of interplanetary parameters causing intense magnetic storms (Dst $<-100$ nT), Planet Space Sci., 35 , $1101-1109,1987$.

Gonzalez, W. D., J. A. Joselyn, Y. Kamide, H. W. Kroehl, G. Rostoker, B. T. Tsurutani, and V. M. Vasyliunas, What is a geomagnetic storm, J. Geophys. Res., 99, 5771-5792, 1994

Gosling, J. T., and D. J. McComas, Field line draping about fast coronal mass ejecta: A source of strong out-of-the-ecliptic interplanetary magnetic fields, Geophys. Res. Lett., 14, 335, 1987.

Gosling, J. T., Coronal mass ejections and magnetic flux ropes in interplanetary space, in Physics of Magnetic Flux Ropes, Geophys. Monogr., vol. 58, edited by C. T. Russell et al., pp. 3518-3528, AGU, Washington, D. C., 1990

Gosling, J. T., D. J. McComas, J. L. Phillips, and J. Bame, Geomagnetic activity associated with earth passage of interplanetary shock disturbances and coronal mass ejections, J. Geophys. Res., 96, 7831-7839, 1991.

Henderson, M. G., G. D. Reeves, and J. S. Murphree, Are north-south aligned auroral structures the ionospheric manifestation of bursty bulk flows?, Geophys. Res. Lett., 25, 3737-3740, 1998.

Henke, T., J. Woch, U. Mall, S. Livi, B. Wilken, R. Schwenn, G. Gloeckler, R. von Steiger, R. J. Forsyth, and A. Balogh, Differences in the $O^{+7} / O^{+6}$ ratio of magnetic cloud and non-cloud coronal mass ejection, 1998

Huttunen, K. E. J., H. E. J. Koskinen, and R. Schwenn, Variability of magnetospheric storms driven by different solar wind perturbations, J. Geophys. Res., 107(D17), 4337, doi:10.1029/2001JD001529, 2002.

Lyons, L. R., Substorms: Fundamental observational features, distinction from other disturbances, and external triggering, J. Geophys. Res., 101, 13,011, 1996.

Lyons, L. R., T. Nagai, G. T. Blanchard, J. C. Samson, T. Yamamoto, T. Mukai, A. Nishida, and S. Kokubun, Association between GEOTAIL plasma flows and auroral poleward boundary intensifications observed by CANOPUS photometers, J. Geophys. Res., 104, 4485, 1999.

Marubashi, K., Interplanetary magnetic flux ropes and solar filaments, in Coronal Mass Ejections, Geophys. Monogr. vol. 99, edited by N. Crooker et al., pp. 147-156, AGU, Washington, D. C., 1997.

McPherron, R. L., The role of substorms in the generation of magnetic storms, in Magnetic Storms, Geophys. Monogr., vol. 98, edited by B. T. Tsurutani et al., pp. 131-147, AGU, Washington, D. C., 1997.

O'Brien, T. P., and R. L. McPherron, Evidence against an independent solar wind density driver of the terrestrial ring current, Geophys. Res. Lett., 27, $3797-3799,2000$
Pulkkinen, A., A. Viljanen, K. Pajunpää, and R. Pirjola, Recordings and occurrence of geomagnetically induced currents in the Finnish natural gas pipeline network, J. Appl. Geophys., 48, 219-231, 2001.

Reeves, G. D., and M. G. Henderson, Ion injections in geosynchronous measurements and composite energetic neutral atom images, J. Geophys. Res., 106, 5833-5844, 2001

Richardson, I. G., and H. V. Cane, Regions of abnormally low proton temperature in the solar wind (1965-1991) and their association with ejecta, J. Geophys. Res., 100, 23,397-23,412, 1995.

Richardson, I. G., E. W. Cliver, and H. V. Cane, Sources of geomagnetic storms for solar minimum and maximum conditions during 1972-2000, Geophys. Res. Lett., 28, 2569-2572, 2001.

Sheeley, N. R., R. A. Howard, M. J. Koomen, D. J. Michels, R. Schwenn, K. H. Mahlhauser, and H. Rosenbauer, Coronal mass ejections and interplanetary shocks, J. Geophys. Res., 90, 163-175, 1985.

Sergeev, V. A., K. Liou, C.-I. Meng, P. T. Newell, M. Brittnacher, G. Parks, and G. D. Reeves, Development of auroral streamers in association with localized impulsive injections to the inner magnetosphere, Geophys. Res. Lett., 26, 417, 1999.

Shue, et al., Magnetopause location under extreme conditions, J. Geophys. Res., 103, 17,691-17,700, 1998.

Tanskanen, E. I., A. Viljanen, T. I. Pulkkinen, R. Pirjola, L. Häkkinen, A. Pulkkinen, and O. Amm, At substorm onset, $40 \%$ of AL comes from underground, J. Geophys. Res., 106, 13,119-13,134, 2001.

Tsurutani, B. T., and W. D. Gonzalez, The interplanetary causes of magnetic storms: A review, in Magnetic Storms, Geophys. Monogr., vol. 98, edited by B. T. Tsurutani et al., pp. 77-89, AGU, Washington, D. C., 1997.

Tsurutani, B. T., and R. P. Lin, Acceleration of $>47 \mathrm{keV}$ ions and $>2 \mathrm{keV}$ electrons by interplanetary shocks at $1 \mathrm{AU}$, J. Geophys. Res., 90, 1-11, 1985.

Tsurutani, B. T., W. D. Gonzalez, F. Tang, S. I. Akasofu, and E. Smith, Origin of interplanetary southward magnetic fields responsible for major magnetic storms near solar maximum (1978-1979), J. Geophys. Res., 93, 8519-8531, 1988 .

Viljanen, A., H. Nevanlinna, K. Pajunpää, and A. Pulkkinen, Time derivative of the horizontal geomagnetic field as an activity indicator, Ann. Geophys., 19, 1107-1118, 2001.

Viñas, A. F., and J. D. Scudder, Fast and optimal solution to the RankineHugoniot Problem, J. Geophys. Res., 91, 39-58, 1986.

Zurbuchen, T. H., S. Hefti, L. A. Fisk, G. Gloeckler, and N. A. Schwadron, Magnetic structure of the slow solar wind: Constraints from composition data, J. Geophys. Res., 105, 18,327, 2000.

Zwickl, R. D., J. R. Asbridge, S. J. Bame, W. C. Feldman, J. T. Gosling, and E. J. Smith, Plasma properties of driver gas following interplanetary shock observed by ISEE-3, Solar Wind Fiver, NASA Conf. Publ., CP$2280,711,1983$.

K. E. J. Huttunen and H. E. J. Koskinen, Department of Physical Sciences, Theoretical Physics Division, University of Helsinki, P.O. Box 64, FIN-00014 Helsinki, Finland. (Emilia.Huttunen@helsinki.fi)

M. Palmroth, A. Pulkkinen, and T. I. Pulkkinen, Geophysical Research, Finnish Meteorological Institute, P.O. Box 503, FIN-00101 Helsinki, Finland.

E. G. D. Reeves, Los Alamos National Laboratory, Los Alamos, NM 87545, USA.

H. J. Singer, NOAA Space Environment Center, 325 Broadway, Boulder, CO 80303, USA. 